J. Math. Soc. Japan

Vol. 24, No. 3, 1972

\title{
On smooth extension theorems
}

\author{
By Hideki OMORI
}

(Received July 24, 1971)

\section{$0^{\circ}$ Introduction.}

It is well known that the space $\boldsymbol{F}$ of the smooth functions of a compact smooth manifold $M$ is a Frechet space and leads a Sobolev chain $\left\{F^{s}\right\}$, namely, $F^{s}$ is a Hilbert space obtained by the completion of $\boldsymbol{F}$ in a norm involving the integral of squares of all derivatives up to order $s$, and $\boldsymbol{F}$ is the inverse limit of the system $\left\{F^{s}\right\}$. Of course the addition $(a, b) \rightarrow a \pm b$ in $\boldsymbol{F}$ can be extended to the smooth mapping of $F^{s} \times F^{s}$ into $F^{s}$ for every $s$.

Assume $M$ is closed (that is, compact without boundary). Then the connected component $\mathscr{D}_{0}$ of the group of the diffeomorphisms of $M$ in $C^{\infty}$-topology has similar properties as above, that is, (1) $\mathscr{D}_{0}$ is a Frechet Lie group [4] and (2) there exists a system $\left\{\mathscr{D}_{0}^{s}\right\}, s \geqq \operatorname{dim} M+5$, of smooth Hilbert manifolds each of which is a topological group such that $\mathscr{D}_{0}$ is the inverse limit of the system $\left\{\mathscr{D}_{0}^{s}\right\} \quad[2,7,9]$. Though the group operations of $\mathscr{D}_{0}^{s}$ is the extension of that of $\mathscr{D}_{0}$, the differentiability of these is not so simple. For example (a) the multiplication $(g, h) \rightarrow g h$ of $\mathscr{D}_{0}$ can be extended to the $C^{l}$-map of $\mathscr{D}_{0}^{s+l} \times \mathscr{D}_{0}^{s}$ into $\mathscr{D}_{0}^{s}$. On the other hand, (b) the right translation $R_{g}: \mathscr{D}_{0}^{s} \rightarrow \mathscr{D}_{0}^{s}$ is smooth for any $g \in \mathscr{D}_{0}^{s}[2,9]$. As a matter of fact, these properties can be proven in case that $M$ has a boundary and $s \geqq \operatorname{dim} M+1$ (c. f. [2]).

In this paper as well as the previous paper [9], the author restricted his concern to the case that (a) $M$ has no boundary, (b) $s \geqq \operatorname{dim} M+5$ and (c) the connected component $\mathscr{D}_{0}$ of the total group of the smooth diffeomorphisms. The reason is the following:

(a) If one constructs an abstract group theory having the properties mentioned above (and this is what he wants to do in the future), then the boundary cases will come in it very naturally.

(b) If $s \geqq \operatorname{dim} M+5$, then we have a nicer property with respect to the composition of maps. Actually, we have a useful inequality in this case (see Theorem A in [9]]).

(c) If one concerns with only local properties, it is enough to treat a neighbourhood of the identity. So as a group generated by a neighborhood of the identity, we have only to consider the connected component $\mathscr{D}_{0}$. 
Anyway, in spite of these various properties of $\mathscr{D}_{0}^{s}$, this seems to be still far from Hilbert Lie groups. Since the tangent space $\Gamma^{s}\left(T_{M}\right)$ of $\mathscr{D}_{0}^{3}$ at the identity. $e$ is not closed under the bracket operation of Lie algebra, we cannot replace the differential structure by some other one so that the group operations may become smooth. Moreover, the properties of $\left\{\mathscr{D}_{0}^{s}\right\}$ mentioned above imply that Frobenius theorem will be fairly difficult to prove on the group $\mathscr{D}_{0}$. Namely, letting $\&$ be a subalgebra of $\Gamma\left(T_{M}\right)$ (the tangent space of $\mathscr{D}_{0}$ at $e$ ), it seems difficult to prove the existence of a topological subgroup of $\mathscr{D}_{0}$ which has $\mathbb{B}$ as a Lie algebra in some sense. As a matter of course, Frobenius. theorem does not hold in the category of Frechet manifolds in general.

However, there is only one way to get a sort of Frobenius theorem on $\mathscr{D}_{0}$. Take the closure $\mathscr{B}^{s}$ of $\mathscr{B}$ in $\Gamma^{s}\left(T_{M}\right)$ and consider the distribution $\widetilde{\mathbb{S}}^{s}=\left\{d R_{\mathrm{g}} \mathbb{G}^{s} ; g \in \mathscr{D}_{0}^{s}\right\}$, where $d R_{\mathrm{g}}$ is the derivative of the right translation $R_{\mathrm{g}}$. In general, this distribution is not differentiable. So suppose this distribution happens to be smooth. Then $\widetilde{\widetilde{S}}^{s}$ is an involutive distribution of $\mathscr{D}_{0}^{s}$ (see Proposition A), where of course $\mathbb{B}$ is provided a subalgebra of $\Gamma\left(T_{M}\right)$. Therefore, one can use the Frobenius theorem in Hilbert manifolds. The resulting manifold $G^{s}$ is a smooth submanifold of $\mathscr{D}_{0}^{3}$ and a subgroup of it. Moreover, the group operation $(g, h) \mapsto g h$ is a $C^{l}$-mapping of $G^{s+l} \times G^{s}$ into $G^{s}$ and the right translation $R_{g}$ is a smooth mapping of $G^{s}$ onto $G^{s}$ for any $g \in G^{s}$. (See also. $2^{\circ}$ for precise statements.)

Therefore the essential thing we have to know is the condition under which $\widetilde{S}^{s}$ becomes differentiable, and the purpose of this paper is to give such conditions which ensure the differentiability of $\mathbb{B}^{3}$. The main result in this paper is the following: Assume $\mathbb{B}$ is the kernel of a differential operator with smooth coefficients and the image of this differential operator is closed. Then $\mathbb{B S}^{s}$ is smooth. (See also $2^{\circ}$ for precise statements.)

As a matter of course, Frobenius theorem on $\mathscr{D}_{0}$ does not finish by the above theorem, because the inverse limit $G$ of $\left\{G^{s}\right\}$ is not necessarily a Frechet manifold. To make $G$ a Frechet manifold, we need much stronger conditions on that differential operator. These will be given in next few papers.

\section{$1^{\circ}$ Review of local properties of groups of diffeomorphisms.}

Before stating the main theorem, we have to establish our notations and recall some results which will be used later. Specifically we discuss local properties of groups of diffeomorphisms. All the proofs of the results in this section may be found in $2^{\circ}$ and $3^{\circ}$ in [9].

Let $\Gamma\left(T_{M}\right), \hat{\Gamma}^{1}\left(T_{M}\right), \hat{\mathscr{D}}^{1}$ denote the space of smooth sections of $T_{M}$ (the 
tangent bundle of $M$ ), the space of $C^{1}$-section of $T_{M}$, the group of $C^{1}$. diffeomorphisms of $M$ with $C^{1}$-topology respectively. Then, there is a homeomorphism $\xi$ of a bounded open neighbourhood $\hat{U}$ of 0 in $\hat{\Gamma}^{1}\left(T_{M}\right)$ onto an open neighbourhood $\tilde{U}$ of the identity in $\hat{\mathscr{D}}^{1}$. Actually this homeomorphism $\xi$ is given by $\xi(u)(x)=\operatorname{Exp}_{x} u(x)$, where $\operatorname{Exp}$ is the exponential mapping defined by a smooth riemannian metric on $M$. Since $\hat{\mathscr{D}}^{1}$ is a topological group, there exists a bounded open neighbourhood $\hat{V}$ of 0 in $\hat{\Gamma}^{1}\left(T_{M}\right)$ such that $\xi(\hat{V})^{2} \subset \tilde{U}$. Put $\boldsymbol{U}=\hat{U} \cap \boldsymbol{\Gamma}\left(T_{M}\right), \boldsymbol{V}=\hat{V} \cap \boldsymbol{\Gamma}\left(T_{M}\right)$. Then, $\eta(u, v)=\xi^{-1}(\xi(u) \xi(v))$ can be regarded as a mapping of $\boldsymbol{V} \times \boldsymbol{V}$ into $\boldsymbol{U}$.

Let $\Gamma^{s}\left(T_{M}\right)$ be the Hilbert space obtained by completion of $\Gamma\left(T_{M}\right)$ in the norm involving the integral of squares of all derivatives up to order $s$. Then the Sobolev embedding theorem shows that if $s \geqq\left[\frac{n}{2}\right]+2, n=\operatorname{dim} M$, then $\Gamma^{s}\left(T_{M}\right) \subset \hat{\Gamma}^{1}\left(T_{M}\right)$ and the inclusion is continuous. So, we put $U^{s}=\hat{U} \cap \Gamma^{s}\left(T_{M}\right)$, $V^{s}=\hat{V} \cap \Gamma^{s}\left(T_{M}\right)$ for $s \geqq\left[\frac{n}{2}\right]+2$.

Using these notations, the properties of $\eta$ are as follows: $(\eta, 1) \eta: V \times V$ $\rightarrow \boldsymbol{U}$ can be extended to the $C^{l}$-mapping of $V^{s+l} \times V^{s}$ into $U^{s}$, where $s \geqq n+5$ and this assumption will keep throughout this paper. $(\eta, 2) \eta_{y}: V^{s} \rightarrow U^{s}$ is a $C^{\infty}$-mapping where $\eta_{y}(x)=\eta(x, y)$. ( $\left.\eta, 3\right)$ Put $\zeta(x, y)=\left(d \eta_{y}\right)_{0} x$, where $\left(d \eta_{y}\right)_{0}$ is the derivative of $\eta_{y}$ at 0 . Then $\zeta: \boldsymbol{\Gamma}\left(T_{M}\right) \times \boldsymbol{V} \rightarrow \boldsymbol{\Gamma}\left(T_{M}\right)$ can be extended to the $C^{l}$-mapping of $\Gamma^{s+l}\left(T_{M}\right) \times V^{s}$ into $\Gamma^{s}\left(T_{M}\right)$. $(\eta, 4)$ There is a neighborhood $\hat{W}$ of 0 in $\hat{V}$ and a continuous map $\phi: \hat{W} \cap \Gamma^{s}\left(T_{M}\right) \rightarrow V^{s}$ such that $\eta(\psi(x), x) \equiv 0$. $(\eta, 5)$ For any $s \geqq n+5$, there is a neighborhood $W^{s}$ of 0 in $V^{s}$ such that for $u, v, v^{\prime} \in W^{s},\left\|\eta\left(v^{\prime}, u\right)-\eta(v, u)\right\|_{s} \leqq K_{s}\left\|v^{\prime}-v\right\|_{s}$ for some constant $K_{s}$. So these properties are regarded as local properties of $\mathscr{D}_{0}^{s}$. Actually the group $\mathscr{D}_{0}^{s}$ is defined as follows: Let $\mathfrak{R}^{s}$ be the basis of neighborhoods of 0 in $\Gamma^{s}\left(T_{M}\right)$. Then, $\left\{\xi(W \cap \boldsymbol{U}) ; W \in \mathfrak{N}^{s}\right\}$ is a family of open neighborhoods of the identity of $\mathscr{D}_{0}$ and defines a new, weaker topology for $\mathscr{D}_{0}$ by which $\mathscr{D}_{0}$ becomes a topological group. So $\mathscr{D}_{0}^{s}$ is the completion of $\mathscr{D}_{0}$ by the (right) uniform topology defined by this new topology. Thus the space $\Gamma^{s}\left(T_{M}\right)$ can be naturally identified with the tangent space at the identity of $\mathscr{D}_{0}^{s}$.

The properties of the system $\left\{\mathscr{D}_{0}, \mathscr{D}_{0}^{s}, s \geqq n+5\right\}$ is the following:

$\left(\mathscr{D}_{0}, 1\right) \quad \mathscr{D}_{0}^{3}$ is a smooth Hilbert manifold.

$\left(\mathscr{D}_{0}, 2\right) \mathscr{D}_{0}^{s+1} \subset \mathscr{D}_{0}^{s}$ and the inclusion is smooth.

$\left(\mathscr{D}_{0}, 3\right) \quad \mathscr{D}_{0}=\cap \mathscr{D}_{0}^{s}$ and the original topology $\left(C^{\infty}\right.$-topology) of $\mathscr{D}_{0}$ is the same as the inverse limit topology.

$\left(\mathscr{D}_{0}, 4\right)$ The multiplication $\mathscr{D}_{0} \times \mathscr{D}_{0} \rightarrow \mathscr{D}_{0},(g, h) \rightarrow g h$, can be extended to the $C^{l}$-mapping of $\mathscr{D}_{0}^{s+l} \times \mathscr{D}_{0}^{s}$ into $\mathscr{D}_{0}^{s}$. 
$\left(\mathscr{D}_{0}, 5\right)$ The mapping $\mathscr{D}_{0} \rightarrow \mathscr{D}_{0}, g \rightarrow g^{-1}$, can be extended to the $C^{l}$-mapping of $\mathscr{D}_{0}^{s+l}$ into $\mathscr{D}_{0}^{s}$.

$\left(\mathscr{D}_{0}, 6\right)$ For any $g \in \mathscr{D}_{0}^{s}$, the right translation $R_{g}: \mathscr{D}_{0}^{s} \rightarrow \mathscr{D}_{0}^{s}$ is smooth.

$\left(\mathscr{D}_{0}, 7\right)$ The mapping $d R: \Gamma^{s+l}\left(T_{M}\right) \times \mathscr{D}_{0}^{s} \rightarrow T \mathscr{D}_{0}^{s}$ defined by $d R(u, g)=d R_{g} u$ is a $C^{l}$-mapping, where $T \mathscr{D}_{0}^{s}$ is the tangent bundle of $\mathscr{D}_{0}^{s}$.

Now, the property $\left(\mathscr{D}_{0}, 7\right)$ can be generalized in the following way: Let $E$ be a smooth riemannian vector bundle and $\boldsymbol{\Gamma}_{g}(E)$ the space of smooth sections. of the pull back $g^{-1} E$ of $E$ by $g \in \mathscr{D}_{0}$. For simplicity, $\Gamma(E)$ denotes the space of smooth sections of $E$ instead of $\boldsymbol{\Gamma}_{i d}(E)$. Consider the set $\gamma(E)=$ $\cup\left\{\boldsymbol{\Gamma}_{g}(E) ; g \in \mathscr{D}_{0}\right\}$. Then $\gamma(E)$ is a vector bundle over $\mathscr{D}_{0}$ in the sense of Frechet manifolds. A local trivialization at the identity is given by the following: Let $C(t), t \in[0,1]$ be a curve in $M$ and $\tau^{\prime}(C)$ the parallel translation along the curve $C$. Of course, $\tau^{\prime}(C)$ is a linear isomorphism of $T_{C(0)} M$ (the tangent space of $M$ at $C(0))$ onto $T_{C(1)} M . \tau^{\prime}(\xi(u)(x)$ ) denotes the parallel. translation along the curve $\operatorname{Exp}_{x} t u(x), t \in[0,1]$. Using this notation, we put $\tau(u, v)(x)=\tau^{\prime}(\xi(u)(x)) v(x)$ for every $u \in U$ and $v \in \Gamma(E)$. As a matter of course, $\tau^{\prime}(\xi(u)(x)) v(x)$ can be regarded naturally as a smooth section of $\Gamma_{\xi(u)}(E)$, if you consider $x$ as a variable. So this $\tau$ makes sense and gives a local trivialization $\tau: \boldsymbol{U} \times \boldsymbol{\Gamma}(E) \rightarrow \gamma(E)$.

On the other hand, for every element $v \in \Gamma_{h}(E), v(g(x))$ can be regarded naturally as an element of $\boldsymbol{\Gamma}_{h g}(E)$ for any $g \in \mathscr{D}_{0}$. So $\mathscr{D}_{0}$ acts (from right) on $\gamma(E)$. This action will be denoted by $R_{g}^{*}$. Obviously $R_{g}^{*} R_{h}^{*}=R_{h g}^{*}$. Putting $\tau_{\xi(u)} v=\tau(u, v)$, the local expression of $R_{\boldsymbol{g}}^{*}$ is given by $\tau_{\xi_{(u) \xi(v)}^{-1}} R_{\xi(v)}^{*} \tau_{\xi(u)}$, where of course $\tau_{\xi(u) \xi(v)}$ implies $\tau_{\xi \eta(u, v) \text {. }}$

Let $R^{\prime}(w, u)=\tau_{\xi(u)}^{-1} R_{\xi(u)}^{*} w, T^{\prime}(u, w, v)=\tau_{\xi(u) \xi(v)}^{-1} R_{\xi(v)}^{*} \tau_{\xi(u)} w$. Then we have

$(\tau, 1) \quad R^{\prime}: \boldsymbol{\Gamma}(E) \times \boldsymbol{U} \rightarrow \boldsymbol{\Gamma}(E)$ can be extended to the $C^{l}$-mapping of $\Gamma^{s+l}(E) \times U^{t}$ into $\Gamma^{s}(E)$ for any $t \geqq s \geqq n+5$.

$(\tau, 2) \quad T^{\prime}: \boldsymbol{V} \times \boldsymbol{\Gamma}(E) \times \boldsymbol{V} \rightarrow \boldsymbol{\Gamma}(E)$ can be extended to the $C^{l}$-mapping of $V^{t+l} \times \Gamma^{s+l}(E) \times V^{t}$ into $\Gamma^{s}(E)$ for any $t \geqq s \geqq n+5$.

In the expression these properties are a little bit different from that of $2^{\circ}$ in [9]. However, these are immediate conclusions of $2^{\circ}[9]$. Because $(\tau, 1)$ $U^{t} \subset U^{s}$ and the inclusion is smooth and $(\tau, 2)$ there is a mapping $\tilde{\tau}_{\boldsymbol{\Delta}}: \boldsymbol{V} \times \boldsymbol{V}$ $\rightarrow \boldsymbol{\Gamma}\left(E^{*} \otimes E\right)$ which can be extended to the smooth mapping of $V^{s} \times V^{s}$ into $\Gamma^{s}\left(E^{*} \otimes E\right)$ (c. f. $\left.2^{\circ}[9]\right)$ such that $T^{\prime}(u, w, v)=\tilde{\tau}_{\Delta}(v, \zeta(u, v)) R^{\prime}(w, v)$, where $\zeta$ is the same mapping as in $\left(r_{l}, 3\right)$. So especially, if $v$ is fixed in $V^{s}$, then $R_{\xi(v)}^{\prime \prime}(w, u)$ defined by $\tau_{\xi(u) \xi(v)}^{-1} R_{\xi(v)}^{*} \tau_{\xi(u)} w$ is a smooth map of $V^{s} \times \Gamma^{s}(E)$ and continuous with respect to $v \in V^{s}$.

Since $\mathscr{D}_{0}^{n+5}$ is a topological group, there exists an open neighbourhood $W$ 
of 0 in $\boldsymbol{\Gamma}^{n+5}\left(T_{M}\right)$ such that $\xi(W) \xi(W)^{-1} \subset \xi\left(V^{n+5}\right)$. Put $\boldsymbol{W}=W \cap \boldsymbol{\Gamma}\left(T_{M}\right), W^{s}=$ $W \cap \Gamma^{s}\left(T_{M}\right)$. Consider the disjoint union $\cup\left\{\xi\left(W^{t}\right) g \times \Gamma^{s}(E) ; g \in \mathscr{D}_{0}^{t}\right\}$. We define an equivalence relation $\sim$ as follows: $(\xi(u) g, w) \sim\left(\xi\left(u^{\prime}\right) g^{\prime}, w^{\prime}\right)$ if and only if $\xi(u) g=\xi\left(u^{\prime}\right) g^{\prime}$ and $w=R_{g^{\prime} g^{-1}}^{\prime \prime}\left(w^{\prime}, u^{\prime}\right)$. Since $g^{\prime} g^{-1} \in \xi\left(V^{t}\right)$, this definition makes sense. So we put $\gamma^{t, s}(E)=\cup\left\{\xi\left(W^{t}\right) g \times \Gamma^{s}(E) ; g \in \mathscr{D}_{0}^{t}\right\} / \sim$. Then, the properties of $\gamma^{t, s}(E)$ are the following:

$(\gamma, 1) \quad \gamma^{t, s}(E)$ is a smooth vector bundle over $\mathscr{D}_{0}^{t}$ with the fibre $\Gamma^{s}(E)$.

$(\gamma, 2) \quad \gamma^{t+1, s}(E) \subset \gamma^{t, s}(E), \gamma^{t, s+1}(E) \subset \gamma^{t, s}(E)$ (where $t \geqq s+1$ ) and the inclusions are smooth.

$(\gamma, 3) \quad \gamma(E)=\cap \gamma^{s}(E)$, where $\gamma^{s}(E)$ is a simplified notation of $\gamma^{s, s}(E)$.

$(\gamma, 4) \quad \gamma^{t, s}(E)$ is the pull back of $\gamma^{s}(E)$ by the inclusion $\mathscr{D}_{0}^{t} \subset \mathscr{D}_{0}^{s}$.

$(\gamma, 5) \quad$ The right translation $R_{g}^{*}$ can be defined for $g \in \mathscr{D}_{0}^{t}$ and is a smooth map of $\gamma^{t, s}(E)$ onto itself. Moreover, this is continuous with respect to $g \in \mathscr{D}_{0}^{t}$.

$(\gamma, 6) \quad$ Put $R^{*}(w, g)=R_{g}^{*} w$ for $w \in \Gamma(E), \quad g \in \mathscr{D}_{0}$. Then this mapping $R^{*}: \Gamma(E) \times \mathscr{D}_{0} \rightarrow \gamma(E)$ can be defined to the $C^{l}$-mapping of $\Gamma^{s+l}(E) \times \mathscr{D}_{0}^{s}$ into $\gamma^{s}(E)$.

These properties are a little bit different from that of $2^{\circ},[9]$ in expression, but all of them are immediate conclusions. Anyway, the properties $(\eta, 1-5)$, $\left(\mathscr{D}_{0}, 1-7\right),(\tau, 1-2)$ and $(\gamma, 1-6)$ are all what we need as a background of this paper.

\section{$2^{\circ}$ Statements of main theorems and applications.}

Let $E, F$ be smooth finite dimensional riemannian vector bundles over $M$. Consider a linear mapping $A: \Gamma(E) \rightarrow \Gamma(E)$ which can be extended to the bounded linear map of $\Gamma^{s}(E)$ into $\Gamma^{s-r}(F)$ for every $s \geqq n+5+r$. Then by using $(\gamma, 5-6)$ above, the right invariant bundle morphism $\tilde{A}: \gamma^{s}(E) \rightarrow \gamma^{s, s-r}(F)$ defined by $R_{g}^{*} A R_{g}^{*-1}$ is continuous. In general, we cannot expect the differentiability of $\tilde{A}$. Moreover, we cannot expect the continuity in operator norm with respect to $g$. Situation is the following:

Suppose $B_{1}, B_{2}$ be smooth Banach vector bundles over a smooth Banach manifold $X$ with fibres $F_{1}, F_{2}$ respectively. Let $\tilde{A}$ be a $C^{k}$-bundle morphism of $B_{1}$ into $B_{2}$. Then, taking local trivializations on some coordinate neighborhood $W, \tilde{A}$ induces a $C^{k}$-map $A^{\prime}$ of $W \times F_{1}$ into $F_{2} . A^{\prime}$ is linear with respect to the second variable. So $A^{\prime}$ induces a mapping $A^{\prime \prime}$ of $W$ into $L\left(F_{1}, F_{2}\right)$. In general, $A^{\prime \prime}$ is only a $C^{k-1}$-mapping. If $A^{\prime}$ is continuous, then for any point $u \in W$, there is a neighborhood $V$ of $u$ and a constant $K$ such 
that $\left\|A^{\prime \prime}(v)\right\| \leqq K$ for any $v \in V$, that is, locally bounded, this is because $A^{\prime}$ is continuous at $(u, 0)$, where \|\|$\|$ is the operator norm. We call $\tilde{A}$ a strong bundle morphism, if $A^{\prime \prime}$ is continuous.

If a bundle morphism $\tilde{A}$ is smooth, there is no confusion, because $A^{\prime}$ and $A^{\prime \prime}$ are smooth. However if $\tilde{A}$ is only continuous, we have to be careful about continuity. We use the notion "continuous" only for the continuity of $A^{\prime}$ as far as concerning bundle morphisms.

Now, we have the following:

THEOREM A (Smooth extension theorem). Suppose $A$ is a differential operator of order $r$ with smooth coefficients. Then $\tilde{A}$ is a smooth right-invariant bundle morphism of $\gamma^{t, s}(E)$ into $\gamma^{t, s-r}(F)$.

This is a main theorem of this paper and a proof will be seen in $4^{\circ}$, using rather known results about jet bundles which will be discussed in $3^{\circ}$. In this section several other simpler extension theorems will be proved, some of which are proved by using this Theorem A.

Now, we have already discussed about some sort of extension theorem, Theorem B [9] in the previous paper. First of all, we have to recollect this theorem. Let $W$ be a relatively compact open subset of $E$ such that $W_{x}$ is a non empty open subset of $E_{x}$ for each $x \in M$, where $E_{x}$ is the fibre of $E$ at $x$ and $W_{x}=W \cap E_{x}$. For any $s \geqq\left[\frac{n}{2}\right]+1$, we put

$$
W^{s}=\left\{u \in \Gamma^{s}(E) ; u(x) \in W_{x} \text { for any } x \in M\right\}
$$

Let $\boldsymbol{W}=\cap W^{s}$. Suppose $f$ is a smooth mapping of $W$ into $F$ such that $f\left(W_{x}\right) \subset F_{x}$ for each $x \in M$. Define a mapping $\Psi: \boldsymbol{W} \rightarrow \boldsymbol{\Gamma}(F)$ by $\Psi(u)(X)=$ $f(u(X))$. Call such $\Psi$ a mapping defined from a smooth function $f$. Then, the simplified Theorem B in [9] shows the following:

$\Psi: \boldsymbol{W} \rightarrow \boldsymbol{\Gamma}(F)$ can be extended to the smooth map of $W^{s}$ into $\Gamma^{s}(F)$.

Now let $1_{M}$ be the trivial vector bundle over $M$ of dimension 1 . The following is easy to prove, by using Sobolev embedding theorem:

LEMMA 1. Let $k_{0}=\left[\begin{array}{l}n \\ 2\end{array}\right]+1$. The mapping $\Phi$ defined by

$$
\Phi(w, v, f)=\int_{M}\langle w(x,), v(x)\rangle_{x} f(x) \mu(x)
$$

is a bounded tri-linear mapping of $\Gamma^{0}(E) \times \Gamma^{0}(E) \times \Gamma^{k_{0}}\left(1_{M}\right)$ into $R$, where $\langle,\rangle_{x}$ is the riemannian inner product of $E$ at $x$ and $\mu$ is a volume element of $M$.

The next lemma is not difficult, since we have the fact but an exact proof will be given in $2^{\circ}$, because we have to use higher order connections for a precise proof.

LEMMA 2. Let $J(u)$ be the Jacobian of $\xi(u)$. Then $J$ is a smooth mapping 
of $U^{k_{0}+1}$ into $\Gamma^{k_{0}}\left(1_{M}\right)$, where $k_{0}=\left[\frac{n}{2}\right]+1$ and $U^{k_{0+1}}$ is the same open set in $\Gamma^{k_{0+1}}(E)$ which is defined in $1^{\circ}$.

Suppose $e_{1}, \cdots, e_{m}$ to be linearly independent elements of $\boldsymbol{\Gamma}(E)$. For any $\gamma^{t, s}(E), t \geqq s \geqq n+5$, we define a mapping $k$ of $\gamma^{t, s}(E)$ into $R^{m}$ by the following:

$$
\begin{aligned}
& k=\left(k_{1}, \cdots, k_{m}\right), \\
& k_{j}\left(R_{g}^{*} w\right)=\int_{M}\left\langle w(x), e_{j}(x)\right\rangle_{x} \mu(x) .
\end{aligned}
$$

THEOREM 1. Notations and assumptions being as above, $k$ is a rightinvariant smooth mapping of $\gamma^{t, s}(E)$ onto $R^{m}$.

PROOF. Surjectivity and right invariance are trivial. So we have only to show the smoothness. By changing the variable $x$, we see

$$
k_{j}\left(R_{g}^{*} w\right)=\int_{M}\left\langle w(g x), e_{j}(g x)\right\rangle_{g x} \mu(g x)
$$

To prove the differentiability, one has to use the local trivialization of $\gamma^{t, s}(E)$ at the identity. So recall the definition of $\tau$ in $1^{\circ}$. Put $R_{\xi(u)}^{*} w^{\prime}=\tau(u, w)$ and we have

$$
k_{j}(\tau(u, w))=\int_{M}\left\langle w^{\prime}(\xi(u)(x)), e_{j}(\xi(u)(x))\right\rangle_{\xi(u)(x)} \mu(\xi(u)(x)),
$$

using (1). Since $w^{\prime}(\xi(u)(x))=\tau^{\prime}(\xi(u)(x)) w(x)$ and $e_{j}(\xi(u)(x))=\tau^{\prime}(\xi(u)(x)) R^{\prime}\left(e_{j}, u\right)(x)$, replacing by these and using the fact that $\tau^{\prime}(\xi(u)(x))$ is an isometry, we get

$$
k_{j}(\tau(u, w))=\int_{M}\left\langle w(x), R^{\prime}\left(e_{j}, u\right)(x)\right\rangle_{x} \mu(\xi(u)(x)) .
$$

Therefore, $k_{j}(\tau(u, w))=\Phi\left(w, R^{\prime}\left(e_{j}, u\right), J(u)\right)$. Since $e_{j} \in \Gamma(E), R^{\prime}\left(e_{j}, u\right)$ is smooth with respect to $u$ (c. f. $(\tau, 2)$ ). So Lemma 2 implies the smoothness of $k$.

The above mapping $k$ can be regarded as a smooth bundle morphism of $\gamma^{t, s}(E)$ onto the trivial bundle $\mathscr{D}_{0}^{t} \times R^{m}$.

LEMMA 3. Let $\tilde{E}, \tilde{F}$ and $\tilde{G}$ be vector bundles over a smooth Hilbert manifold $X$ with the fibres $E, F$ and $G$ of Hilbert spaces respectively. Suppose $\tilde{E}$ and $\widetilde{F}$ are smooth bundles. Let $\tilde{A}: \widetilde{E} \rightarrow \widetilde{F}$ be a smooth bundle morphism and $\tilde{B}: \widetilde{F} \rightarrow \tilde{G}$ a strong bundle morphism such that $\operatorname{Im} \tilde{A}=\operatorname{Ker} \widetilde{B}$ and $\tilde{B}$ is surjective. Then $\operatorname{Ker} \tilde{A}$ is a smooth subbundle of $\tilde{E}$.

PROOF. Fix an arbitrary point $x \in X$. There is a neighbourhood $W$ of $x$ such that $\tilde{E}|W \cong W \times E, \widetilde{F}| W \cong W \times F$ and $\tilde{G} \mid W \cong W \times G$. Of course, $\tilde{E} \mid W$ etc. implies the restriction of $\widetilde{E}$ on $W$ and $\cong$ does local trivialization. Thus, $\tilde{A}$ induces a smooth map $A^{\prime}: W \times E \rightarrow F$ and $\tilde{B}$ does a strong map $B^{\prime}: W \times F \rightarrow G$, each of which is linear with respect to the second variable. Let $E_{1}=\operatorname{Ker} A_{x}^{\prime}$, $F_{1}=\operatorname{Im} A_{x}^{\prime}=\operatorname{Ker} B_{x}^{\prime}$, where $A_{y}^{\prime} v=A^{\prime}(y, v), B_{y}^{\prime} v=B^{\prime}(y, v)$. Let $E_{2}$ (resp. $\left.F_{2}\right)$ be the orthogonal complement of $E_{1}$ (resp. $F_{1}$ ). 
Since $B_{x}^{\prime}: F_{2} \rightarrow G$ and $A_{x}^{\prime}: E_{2} \rightarrow F_{1}$ are isomorphisms, letting $\pi$ be the projection of $F$ onto $F_{1}$, we can take $W$ so small that it may satisfy $B_{y}^{\prime}: F_{2} \rightarrow G$, $\pi A_{y}^{\prime}: E_{2} \rightarrow F_{1}$ are still isomorphisms for any $y \in W$. Take such $W$. We make a new smooth mapping $C: W \times E \oplus F_{2} \rightarrow F$ by $C_{y}(u+v)=A_{y}^{\prime} u+v$. It is easy to see that $C_{y}$ is surjective for any $y \in W$. If $C_{y}(u+v)=0$, then $\pi A_{y}^{\prime} u=0$ and $(1-\pi) A_{y}^{\prime} u+v=0$. Therefore $A_{y}^{\prime} u=0$, because if not, then $A_{y}^{\prime} u \in F_{2}$, hence $B_{y}^{\prime} A_{y}^{\prime} u \neq 0$, contradicting the assumptions. So, $C_{y}(u+v)=0$ yields $u \in \operatorname{Ker} A_{y}^{\prime}$ and $v=0$. Consequently, we get $\operatorname{Ker} C_{y}=\operatorname{Ker} A_{y}^{\prime}$. Thus, this lemma is reduced to the case $\tilde{G}=\{0\}$, that is, $\tilde{A}$ is surjective.

So put $F=F_{1}, F_{2}=\{0\}$. We can find $W$ such that $A_{y}^{\prime}$ is an isomorphism of $E^{2}$ onto $F$ for every $y \in W$. Let $D_{y}$ be the inverse of $A_{y}: E_{2} \rightarrow F . D_{y}$ is smooth with respect to $y$. Put $\Psi: W \times E_{1} \rightarrow E$ by $\Psi(y, u)=u-D_{y} A_{y}^{\prime} u$. Obviously, $A_{y}^{\prime} \Psi(y, u) \equiv 0$, and $\Psi$ is a smooth mapping. So this $\Psi$ can be regarded as a local trivialization of $\operatorname{Ker} \tilde{A}$, because if $A_{y}^{\prime} u=0$ for some $(y, u)$, then letting $\pi^{\prime}: E \rightarrow E_{1}$ be the projection, $u=\pi^{\prime} u-D_{y} A_{y}^{\prime} \pi^{\prime} u$. This relation is gotten by the following: $\pi^{\prime} u-D_{y} A_{y}^{\prime} \pi^{\prime} u$ is contained in $\operatorname{Ker} A_{y}^{\prime}$. So if $D_{y} A_{y}^{\prime} \pi^{\prime} u=$ $\left(1-\pi^{\prime}\right) u$, then the difference $\left(1-\pi^{\prime}\right) u-D_{y} A_{y}^{\prime} \pi^{\prime} u$ is contained in $E_{2} \cap \operatorname{Ker} A_{y}^{\prime}$, which is $\{0\}$ by assumption.

THEOREM 2. Suppose $A: \boldsymbol{\Gamma}(E) \rightarrow \boldsymbol{\Gamma}(F)$ is a differential operator of order $r$ with smooth coefficients and assume the image $A \Gamma^{s}(E)$ is closed in $\Gamma^{s-r}(F)$ for any $s \geqq n+5+r$. Then, for any $t \geqq s$, Ker $\tilde{A}$ is a right invariant smooth subbundle of $\gamma^{-, s}(E)$.

Proof. Suppose at first that the image $\operatorname{Im} \tilde{A}$ is a continuous subbundle. Then, the factor bundle $\gamma^{t, s-r}(F) / \operatorname{Im} \tilde{A}$ is a continuous vector bundle and

$$
\gamma^{t, s}(E) \stackrel{\tilde{A}}{\longrightarrow} \gamma^{t, s-r}(F) \stackrel{\tilde{\pi}}{\longrightarrow} \gamma^{t, s-r}(F) / \operatorname{Im} A \longrightarrow 0
$$

is exact, where $\tilde{\pi}$ is the natural projection. Since the factor bundle is continuous, $\tilde{\pi}$ is a strong bundle morphism. Therefore, Theorem A together with the above Lemma yields the desired result.

Therefore, we have only to show that $\operatorname{Im} \tilde{A}$ is a continuous subbundle of $\gamma^{t, s-r}(F)$. Take local trivializations $\tau: U^{t} \times \Gamma^{s}(E) \rightarrow \gamma^{t, s}(E)$ and $\tau: U^{t} \times \Gamma^{s-r}(F)$ $\rightarrow \gamma^{t, s-r}(F)$ as in $1^{\circ}$. Then, $\tilde{A}$ induces a smooth map $A^{\prime}: U^{t} \times \Gamma^{s}(E) \rightarrow \Gamma^{s-r}(F)$. $A^{\prime}$ is linear with respect to the second variable. Let $E_{1}=\operatorname{Ker}\left\{A: \Gamma^{s}(E) \rightarrow\right.$ $\left.\Gamma^{s-r}(F)\right\}, F_{1}=A \Gamma^{s}(E)$ and $E_{2}, F_{2}$ orthogonal complements of $E_{1}, F_{1}$ respectively. Then, $A: E_{2} \rightarrow F_{1}$ is an isomorphism. Let $G$ be its inverse, and the map $\varphi: U^{t} \times F_{1} \rightarrow F_{1} \oplus F_{2}$ defined by $\varphi(u, v)=A^{\prime}(u, G v)$ is smooth and satisfies $\varphi(0, v) \equiv v$. Let $\pi$ be the projection of $F_{1} \oplus F_{2}$ onto $F_{1}$. Then, there is a neighborhood $W$ of 0 in $U^{\iota}$ such that $\pi \varphi_{u}$ defined by $\pi \varphi_{u} v=\pi \varphi(u, v)$ is an isomorphism for any $u \in W$. Therefore the image $\cup\left\{\varphi_{u} F_{1} ; u \in W\right\}$ defines a smooth subbundle of $W \times F_{1} \oplus F_{2}$, where $\varphi_{u} v=\varphi(u, v)$. Thus, we have only to 
show $\varphi_{u} F_{1}=A_{u}^{\prime} \Gamma^{s}(E)$ if $u$ is sufficiently near to 0 , where $A_{u}^{\prime} v=A^{\prime}(u, v)$.

So assume there were a sequence $\left\{x_{n}\right\}$ converging to 0 in $U^{t}$ such that $A_{x_{n}}^{\prime} \Gamma^{s}(E) \supsetneq A_{x_{n}}^{\prime} E_{2}$. Then, there exist $u_{n}$ such that $A_{x_{n}}^{\prime} u_{n} \in F_{2}$ and $\left\|A_{x_{n}}^{\prime} u_{n}\right\|_{s-r}$ $=1$ for sufficiently large $n$. We may assume $u_{n} \in \tau{\widetilde{\xi}\left(x_{n}\right)}^{-1} R_{\xi\left(x_{n}\right)}^{*} E_{2}$. Let $y_{n}=$ $\xi^{-1}\left(\xi\left(x_{n}\right)^{-1}\right) . \quad\left\{y_{n}\right\}$ converges to 0 in $U^{t}$ (c. f. $\left.(\eta, 4)\right)$. Since $R^{\prime}(v, x)\left(=\tau \bar{\xi}_{(x)}^{-1} R_{\xi(x)}^{*} v\right)$ is a continuous mapping of $\Gamma^{s}(E) \times U^{t}$ into $\Gamma^{s}(E)$, there are a neighbourhood $W$ of 0 in $U^{t}$ and $K$ such that $\left\|\tau_{\xi(x)}^{-1} R_{\xi(x)}^{*}\right\| \leqq K$ for any $x \in W$. Namely, $\left\|\tau \bar{\xi}^{-1}(x) R_{\xi(x)}^{*} v\right\|_{s} \leqq K\|v\|_{s}$ for any $x \in W$. Since $\tau_{\xi\left(y_{n}\right)}^{-1} R_{\xi(y n)}^{*} \tau_{\xi\left(x_{n}\right)}^{-1} R_{\xi\left(x_{n}\right)}^{*}=\mathrm{id}$., we have

$$
K^{-1}\|v\|_{s} \leqq\left\|\tau{\bar{\xi}\left(x_{n}\right)}^{-1} R_{\xi\left(x_{n}\right)}^{*} v\right\|_{s} \leqq K\|v\|_{s}, \quad v \in \Gamma^{s}(E),
$$

for sufficiently large $n$, and similarly we have

$$
K^{-1}\|v\|_{s-r} \leqq\left\|\tau_{\xi\left(x_{n}\right)}^{-1} R_{\xi\left(x_{n}\right)}^{*} v\right\|_{s-r} \leqq K\|v\|_{s-r}, \quad v \in \Gamma^{s-r}(F) .
$$

Since $A_{x_{n}}^{\prime}=\tau_{\xi\left(x_{n}\right)}^{-1} \tilde{A} \tau_{\xi\left(x_{n}\right)}$, we have

$$
A_{x_{n}}^{\prime} u_{n}=\tau_{\xi\left(x_{n}\right)}^{-1} R_{\xi\left(x_{n}\right)}^{*} A \tau_{\xi\left(y_{n}\right)}^{-1} R_{\xi\left(y_{n}\right)}^{*} u_{n} .
$$

Therefore, there is a constant $C$ such that $\left\|A_{x_{n}}^{\prime} u_{n}\right\|_{s-r} \geqq C\left\|u_{n}\right\|_{s}$, namely $\left\{u_{n}\right\}$ is bounded. Notice that $\lim A_{x_{n}}^{\prime}=A_{0}^{\prime}$ in operator norm of $L\left(\Gamma^{s}(E), \Gamma^{s-r}(F)\right.$ ). Put $u_{n}=v_{n}+w_{n}, v_{n} \in E_{1}, w_{n} \in E_{2}$. Then $\left\{v_{n}\right\},\left\{w_{n}\right\}$ are also bounded. Therefore,

$$
\begin{aligned}
1 & \equiv \lim \left\|A_{x_{n}}^{\prime} u_{n}\right\|_{s-r} \leqq \lim \left\|A_{x_{n}}^{\prime} w_{n}\right\|_{s-r}+\lim \left\|\left(A_{x_{n}}^{\prime}-A_{0}^{\prime}\right) v_{n}\right\|_{s-r} \\
& =\lim \left\|A_{x_{n}}^{\prime} w_{n}\right\|_{s-r} \leqq \lim \left\|A_{0}^{\prime} w_{n}\right\|_{s-r}+\lim \left\|\left(A_{x_{n}}^{\prime}-A_{0}^{\prime}\right) w_{n}\right\|_{s-r} \\
& =\lim \left\|A_{0}^{\prime} w_{n}\right\|_{s-r} \leqq \lim \left\|\pi A_{x_{n}}^{\prime} w_{n}\right\|_{s-r}+\lim \left\|\pi\left(A_{x_{n}}^{\prime}-A_{0}^{\prime}\right) w_{n}\right\|_{s-r} \\
& =\lim \left\|\pi A_{x_{n}}^{\prime} w_{n}\right\|_{s-r} \leqq \lim \left\|\pi A_{x_{n}}^{\prime} u_{n}\right\|_{s-r}+\lim \left\|\pi\left(A_{x_{n}}^{\prime}-A_{0}^{\prime}\right) v_{n}\right\|_{s-r} \\
& =\lim \left\|\pi A_{x_{n}} u_{n}\right\|_{s-r} \equiv 0 .
\end{aligned}
$$

This is contradiction, hence there exists a neighborhood $W$ of 0 in $U^{t}$ such that $\varphi_{x} F_{1}=A_{x}^{\prime} E_{2}=A_{x}^{\prime} \Gamma^{s}(E)$ for any $x \in W$.

Now let $\&$ be a subalgebra of $\Gamma\left(T_{M}\right)$. Let $\mathscr{S}^{s}$ be the closure of $\mathbb{S}$ in $\Gamma^{s}\left(T_{M}\right)$. Put $\widetilde{G}^{s}=\left\{d R_{g} \mathbb{S}^{s} ; g \in \mathscr{D}_{0}^{s}\right\}$. In general, this is not a continuous distribution (i. e. continuous subbundle).

Proposition A. Notations being as above, suppose $\widetilde{\mathfrak{G}}^{s}$ is smooth. Then $\widetilde{\mathfrak{G}}^{s}$ is involutive. (This condition is satisfied, if $\mathbb{B S}$ is defined by a kernel of a differential operator satisfying the assumption of Theorem 2 or by a kernel of $k$ in Theorem 1.) More generally, if $\widetilde{\mathbb{S}}^{s}$ is a $C^{k(s)}$-distribution for some $k(s) \geqq 1$ depending on $s$, then $\widetilde{G}^{s}$ is involutive.

ProOF. First of all, we fix $s$ arbitrarily. Since $\widetilde{G} s$ is a $C^{k(s)}$-distribution on a smooth Hilbert manifold $\mathscr{D}_{0}^{s}$, there is so called $\widetilde{\mathscr{S}}^{s}$-connection $\nabla$ on $\mathscr{D}_{0}^{s}$,

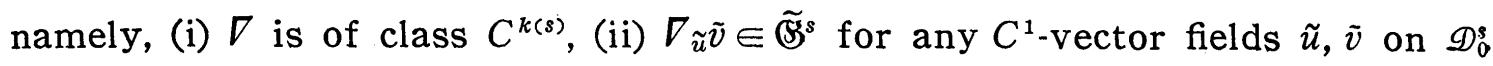


contained in $\widetilde{\mathscr{S}}^{s}$. The existence of such connection is not hard to prove, if one uses a partition of unity. Moreover, in this case, we need such a connection defined only on a neighborhood of the identity. So in fact, one can use a local coordinate and a local trivialization for the definition of $\nabla$.

Put $T(\tilde{u}, \tilde{v})=\nabla_{\tilde{u}} \tilde{v}-\nabla_{\tilde{v}} \tilde{u}-[\tilde{u}, \tilde{v}]$. Then, $T$ is called a torsion tensor and a tensor field on the neighborhood of the identity. Put $\tilde{u}(g)=d R_{g} u$ for $u \in \mathbb{S}$. Then, $\tilde{u}$ is a smooth vector field on $\mathscr{D}_{0}^{s}$ contained in $\widetilde{\mathbb{S}}^{s}$ and $[\tilde{u}, \tilde{v}](g)=d R_{g}[u, v]$ for any $u, v \in \mathbb{S}$. Therefore, $T\left(d R_{g} u, d R_{g} v\right) \in d R_{g} \mathbb{S}^{s}$ for any $u, v \in \mathbb{S}$. This implies $T\left(d R_{g} \mathbb{G}^{s}, d R_{g} \mathbb{G}^{s}\right) \subset d R_{g} \mathbb{G}^{s}$ because $T$ is a bounded bilinear map at each point. Thus, we have the torsion tensor satisfies $T\left(\widetilde{\mathscr{S}}^{s}, \widetilde{\mathscr{S}}^{s}\right) \subset \widetilde{\mathscr{S}}^{s}$. This implies $\widetilde{\mathbb{S}}^{s}$ is involutive.

Now suppose $\widetilde{\mathscr{S}}^{s}=\left\{d R_{g} \mathscr{S}^{s} ; g \in \mathscr{D}_{0}^{s}\right\}$ is a smooth distribution of $\mathscr{D}_{0}^{s}$ for $s \geqq s_{0}$, where $s_{0}$ is some positive integer $\geqq n+5$. By using Frobenius theorem on Hilbert manifolds, there exists the maximal integral submanifold $G^{s}$ through the identity. Put $G=\cap G^{s}$. Then $\left\{G, G^{s}\right\}$ satisfies the following properties:

$(G, 1) \quad G^{s}$ is a smooth Hilbert manifold (trivial).

$(G, 2) \quad G^{s+1} \subset G^{s}$ and the inclusion is smooth. (See below.)

$(G, 3) \quad G=\cap G^{s}$ (by definition). We give the inverse limit topology on $G$.

$(G, 4) \quad G$ is a topological group by the inverse limit topology and the group multiplication $G \times G \rightarrow G,(g, h) \rightarrow g h$, can be extended to the $C^{l}$-mapping of $G^{s+l} \times G^{s}$ into $G^{s}$. (See below.)

$(G, 5)$ The mapping $G \rightarrow G, g \rightarrow g^{-1}$, can be extended to the $C^{l}$-mapping of $G^{s+l}$ into $G^{s}$. (See below.)

$\{G, 6)$ For any $g \in G^{s}$, the right translation $R_{g}: G^{s} \rightarrow G^{s}$ is smooth. (This is because $\widetilde{\mathscr{S}}^{s}$ is right invariant and $R_{\mathrm{g}}: \mathscr{D}_{0}^{s} \rightarrow \mathscr{D}_{0}^{s}$ is smooth.)

$(G, 7) \quad$ The mapping $d R: \mathbb{S}^{s+4} \times G^{s} \rightarrow T G^{s}$ defined by $d R(u, g)=d R_{g} u$ is $C^{l}$. mapping, where $T G^{s}$ is the tangent bundle of $G^{s}$. (See below.)

To prove these properties $G, 2.4 .5 .7$, we need a lemma.

LEMMA 4. $G^{s}$ is a topological group for any $s \geqq s_{0}$.

PROOF. Since $\widetilde{\mathscr{S}}^{s}$ is invariant, we have $R_{g} G^{s}=G^{s}$ for any $g \in G^{s}$. This shows $G^{s}$ is a subgroup. So $G^{s}$ is a topological group by the relative topology. However, the manifold structure might be given by a stronger topology, because $G^{s}$ might not be closed in $\mathscr{D}_{0}^{s}$.

Now, let $E^{s}$ be the orthogonal complement of $\mathbb{B}^{s}$ in $\Gamma^{s}\left(T_{M}\right)$. Therefore there are open neighbourhoods $V^{s}, W^{s}$ of 0 in $\mathbb{B}^{s}, E^{s}$ respectively and a smooth diffeomorphism $\xi_{s}^{\prime}$ of $V^{s} \times W^{s}$ onto an open neighbourhood of the identity such that $\xi_{s}^{\prime}\left(V^{s}, u\right)$ is an integral manifold of $\widetilde{\mathscr{S}}^{s}$ for every $u \in W^{s}$. Since $\mathscr{D}_{0}^{s}$ is a 
topological group, there are neighbourhoods $\bar{V}^{s}, W^{s}$ of 0 in $\mathbb{S}^{s}, E^{s}$ respectively such that $\xi_{s}^{\prime}\left(\bar{V}^{s}, \hat{W}^{s}\right) \xi_{s}^{\prime}\left(\bar{V}^{s}, W^{s}\right)^{-1} \subset \xi_{s}^{\prime}\left(\bar{V}^{s}, \bar{W}^{s}\right)$. This implies $\xi_{s}^{\prime}\left(\bar{V}^{s}, 0\right) \xi_{s}^{\prime}\left(\bar{V}^{s}, 0\right)^{-1} \subset$ $\xi_{s}^{\prime}\left(\bar{V}^{s}, 0\right)^{-1} \subset \xi_{s}^{\prime}\left(\bar{V}^{s}, 0\right)$.

On the other hand, it is easy to see that $G^{s}$ is generated by any open neighbourhood $\xi_{s}^{\prime}\left(V^{s}, 0\right)$ of the identity, because $G^{s}$ is connected. Let $\mathfrak{R}^{s}$ be a basis of the neighbourhoods of the identity in $\mathbb{S}^{s}$. Then the system $\left\{\xi_{s}^{\prime}\left(V^{s}, 0\right) ; V^{s} \in \mathfrak{R}^{s}\right\}$ satisfies the axiom of the neighbourhood of topological groups. (As for the axioms, see [11] or [7] for example.) Thus, $G^{s}$ is a topological group.

To prove $(G, 2)$, we have to take two diffeomorphisms $\xi_{s+1}^{\prime}, \xi_{s}^{\prime}$. Assume $\xi_{s+1}^{\prime}\left(V^{s+1} \times W^{s+1}\right) \subset \xi_{s}^{\prime}\left(V^{s} \times W^{s}\right)$. Since $\xi_{s+1}^{\prime}\left(V^{s+1}, 0\right)$ is an integral submanifold of $\widetilde{\mathbb{S}}^{s+1}$ and $\widetilde{\mathscr{S}}^{s+1} \subset \widetilde{\mathscr{S}}^{s}$, any curve $c(t)$ contained in $V^{s+1}$ satisfies $\frac{d}{d t} \xi_{s+1}^{\prime} c(t) \in \widetilde{\mathscr{S}}^{s+1}$ $\subset \widetilde{\mathscr{S}}^{s}$. Thus, $\xi_{s}^{\prime} c(t)$ is contained in the integral submanifold of $\widetilde{\mathscr{S}}^{s}$. This implies $\xi_{s+1}^{\prime}\left(V^{s+1}, 0\right) \subset \xi_{s}^{\prime}\left(V^{s}, 0\right)$. Thus $G^{s+1} \subset G^{s}$.

Together with Lemma 4, the property $(G, 2)$ implies that $G$ is a topological group by the inverse limit topology.

To get the property $(G, 4)$, we take also $\xi_{s+l}^{\prime}, \xi_{s}^{\prime}$ such that

$$
\xi_{s+l}^{\prime}: V^{s+l} \times W^{s+l} \longrightarrow \mathscr{D}_{0}^{s+l}, \quad \xi_{s}^{\prime}: V^{s} \times W^{s} \longrightarrow \mathscr{D}_{0}^{s}
$$

give smooth charts. Since $\mathscr{D}_{0}^{s}$ is a topological group, there are neighborhoods $\bar{V}^{s+l}, \bar{V}^{s}$ such that $\xi_{s+l}^{\prime}\left(\bar{V}^{s+l}, 0\right) \xi_{s}^{\prime}\left(\bar{V}^{s}, 0\right) \subset \xi_{s}^{\prime}\left(V^{s}, W^{s}\right)$. It is easy to see that $\xi_{s+l}^{\prime}\left(\bar{V}^{s+l}, 0\right) \xi_{s}^{\prime}\left(\bar{V}^{s}, 0\right) \subset \xi_{s}^{\prime}\left(V^{s}, 0\right)$. So by the property $\left(\mathscr{D}_{0}, 4\right), \quad \eta_{s, l}(u, v)=$ $\xi_{s}^{\prime-1} \xi_{s+l}^{\prime}(u, 0) \xi_{s}^{\prime}(v, 0)$ is a $C^{1}$-mapping.

By the similar method, we get $(G, 5.7)$.

Now, the properties $(G 1-7)$ is the same to $\left(\mathscr{D}_{0} 1-7\right)$. According to the terminology in $[2,7], G$ is called an I.L.H.-Lie subgroup of $\mathscr{D}_{0}$. In fact, a topological group $G$ is called an I.L.H.-Lie group, if $G$ leads a system $G^{s}$ which satisfy $\left(\mathscr{D}_{0} 1-7\right)$ and an I. L. H.-Lie group $H$, leading a system $H^{s}$, is called an I.L.H.-Lie subgroup of $G$, if $H \subset G, H^{s} \subset G^{s}$, the inclusion is smooth and $\mathscr{S}^{s}$ (the tangent space of $H^{s}$ at $e$ ) is a closed subspace of $\mathbb{S}^{s}$. Strictly speaking, one should call $\left\{G, G^{s}\right\}$ an I. L. H.-Lie group or an I. L. H.-Lie subgroup instead of $G$, because there might exist many kinds of systems $\left\{G^{s}\right\}$ such that $G=\cap G^{s}$. So the system $\left\{G^{s}\right\}$ can be regarded as a structure (that is, an I. L. H.-structure) of $G$. Therefore, when we say $G$ is an I. L. H.-Lie group, we always consider a group $G$ with an I. L. H.-structure $\left\{G^{s}\right\}$. It is easy to see that if $G$ is an I.L.H.-Lie group, then $\mathbb{B}=\cap \mathbb{S}^{s}$ is a Lie algebra, where $\mathscr{G}^{s}$ is the tangent space of $G^{s}$ at $e$ (c.f. $[2,7]$ ). This will be called a Lie algebra of $G$.

COROLLARY 1. Let $\mathbb{B}$ be a subalgebra of $\Gamma\left(T_{M}\right)$ defined by a kernel of a differential operator of order $r$. Let $A: \Gamma\left(T_{M}\right) \rightarrow \Gamma(E)$ denote the differential 
operator. Suppose (i) the coefficients of $A$ are smooth and (ii) the image $A \Gamma^{s}\left(T_{M}\right)$ is closed in $\Gamma^{s-r}(E)$ for any $s \geqq n+5+r$. Then, there exists an I.L.H.-Lie subgroup $G$ of $\mathscr{D}_{0}$ having $\mathbb{B}$ as a Lie algebra.

This is an immediate conclusion of Proposition A and Theorem 2. However, Theorem 2, itself is an immediate conclusion of Theorem A and Lemma 3, and this is not only one conclusion. One can extend Theorem 2 variously. In the following part of this section, a sort of extension of Theorem 2 will be discussed.

Suppose $M$ is oriented and let $E_{-1}, E_{0}, E_{1}$ be finite dimensional smooth riemannian vector bundles over $M$. Suppose $A: \boldsymbol{\Gamma}\left(E_{-1}\right) \rightarrow \boldsymbol{\Gamma}\left(E_{0}\right)$ and $B: \boldsymbol{\Gamma}\left(E_{0}\right)$ $\rightarrow \Gamma\left(E_{1}\right)$ be linear differential operators of order $r$ such that (i) $A$ and $B$ have smooth coefficients, (ii) $B A=0$ and (iii) $A A^{*}+B^{*} B$ is elliptic, where $A^{*}, B^{*}$ are formal adjoint operators of $A, B$ respectively. Let $\square$ denote the elliptic operator $A A^{*}+B^{*} B$. Let $H$ be the kernel of $\square: \Gamma^{2 r}\left(E_{0}\right) \rightarrow \Gamma^{0}\left(E_{0}\right)$. Then, $H$ is finite dimensional and $H \subset \Gamma\left(E_{0}\right)$ by the regularity of the solutions. Define a subbundle $\widetilde{H}^{s}$ by $\left\{R_{g}^{*} H ; g \in \mathscr{D}_{0}^{s}\right\}$. Then $\widetilde{H}^{s}$ is a smooth subbundle of $\gamma^{s}\left(E_{0}\right)$ by the property $(r, 5-6)$. Moreover $\tilde{H}^{t}$ is a smooth subbundle of $\gamma^{t, s}\left(E_{0}\right)$ for any $t \geqq s \geqq n+5$.

Since $H$ is finite dimensional, we can define the right invariant smooth mapping $K$ of $\gamma^{t, s}\left(E_{0}\right)$ onto $H$ by Theorem 1 . Let $\gamma_{a}^{t, s}\left(E_{0}\right)$ be the kernel of $K$. Then by Lemma 3, $\gamma_{0}^{t, s}\left(E_{0}\right)$ is a smooth subbundle of $\gamma^{t, s}\left(E_{0}\right)$ and is right invariant. Let $\widetilde{\square}=R_{\boldsymbol{g}}^{*} \square R_{\boldsymbol{g}}^{*-1}$. Then by using Theorem $\mathrm{A}$, $\widetilde{\square}$ is a smooth bundle morphism of $\gamma^{s}\left(E_{0}\right)$ into $\gamma^{s, s-2 r}\left(E_{0}\right)$ for any $s \geqq n+5+2 r$. Since $M$ is closed, we have the following

LEMMA 5. $\widetilde{\square}$ is a smooth bundle isomorphism of $\gamma_{\square}^{s}\left(E_{0}\right)$ onto $\gamma_{\square}^{s, s-2 r}\left(E_{0}\right)$. Therefore $\widetilde{\square}^{-1}: \gamma_{\square}^{s, s-2 r}\left(E_{0}\right) \rightarrow \gamma_{\square}^{s}\left(E_{0}\right)$ is also smooth.

PROOF. Since $\widetilde{\square}: \gamma_{\square}^{s}\left(E_{0}\right) \rightarrow \gamma_{\square}^{s, s-2 r}\left(E_{0}\right)$ is smooth and injective, we have only to see that the image of $\widetilde{\square}$ is $\gamma^{s, s-2 r}\left(E_{0}\right)$, because if so, it is easy to prove the smoothness of $\widetilde{\square}^{-1}$.

Let $\Gamma_{\square}^{t}\left(E_{0}\right)=\left\{u \in \Gamma^{t}\left(E_{0}\right) ; \int_{M}\langle u, e\rangle \mu=0\right.$ for any $\left.e \in H\right\}$. Then $\Gamma_{\square}^{s}\left(E_{0}\right)$, $\Gamma_{\square}^{s-2 r}\left(E_{0}\right)$ are the fibres at the identity of $r_{c}^{s}\left(E_{0}\right), \gamma_{0}^{s, s-2 r}\left(E_{0}\right)$ respectively. Since $\square$ is self-adjoint elliptic operator, we see $\square: \Gamma_{\square}^{\mathrm{s}}\left(E_{0}\right) \rightarrow \Gamma_{\mathrm{a}}^{\mathrm{s}-2 r}\left(E_{0}\right)$ is isomorphic. Thus, $\widetilde{\square}$ is an isomorphism of $\gamma_{\square}^{s}\left(E_{0}\right)$ onto $\gamma_{\square}^{8,8-2 r}\left(E_{0}\right)$. The smoothness comes from Theorem A.

It is easy to see that $\widetilde{\square} \gamma_{\square}^{s}\left(E_{0}\right)=\gamma_{\square}^{s, s-2 r}\left(E_{0}\right)=\widetilde{\square} \gamma^{s}\left(E_{0}\right)$. Let $\boldsymbol{F}_{1}=\operatorname{Ker} B \cap$ $\boldsymbol{\Gamma}_{\square}\left(E_{0}\right), \quad \boldsymbol{F}_{2}=\operatorname{Ker} A^{*} \cap \boldsymbol{\Gamma}_{\square}\left(E_{0}\right), \quad F_{1}^{s}=\operatorname{Ker} B \cap \Gamma_{\square}^{s}\left(E_{0}\right)$ and $F_{2}^{s}=\operatorname{Ker} A^{*} \cap \Gamma_{\square}^{s}\left(E_{0}\right)$. Then, $F_{1}^{s}, F_{2}^{s}$ are closed in $\Gamma_{\mathrm{d}}^{s}\left(E_{0}\right)$. Such $F_{i}^{s}$ is defined for $s \geqq r$.

LeMMA 6. $\boldsymbol{\Gamma}_{\mathrm{\square}}\left(E_{0}\right)=\boldsymbol{F}_{1} \oplus \boldsymbol{F}_{2}, \Gamma_{\square}^{s}\left(E_{0}\right)=F_{1}^{s} \oplus F_{2}^{s}$ for $s \geqq r$ and $F_{1}^{s}=A \Gamma^{s+r}\left(E_{-1}\right)$, $F_{2}^{s}=B^{*} \Gamma^{s+r}\left(E_{1}\right)$. Moreover, $B \Gamma^{s+r}\left(E_{0}\right)$ is closed in $\Gamma^{s}\left(E_{1}\right)$ and so is $A^{*} \Gamma^{s+r}\left(E_{0}\right)$ 
in $\Gamma^{s}\left(E_{-1}\right)$ for $s \geqq 2 r$.

ProOF. Since $\left\{u \in \Gamma^{s}\left(E_{0}\right) ; A^{*} u=B u=0\right\}=H$ for $s \geqq r$, we see that $F_{1}^{s} \cap F_{2}^{s}=\{0\}$. Since $A A^{*} \square^{-1} u \in F_{1}^{s}, B^{*} B \square^{-1} u \in F_{2}^{s}$, the equality $u=A A^{*} \square^{-1} u$ $+B^{*} B \square^{-1} u$ implies $\Gamma_{\square}^{s}\left(E_{0}\right)=F_{1}^{s} \oplus F_{2}^{s}$ for $s \geqq r$. Hence $\Gamma_{\square}\left(E_{0}\right)=\boldsymbol{F}_{1} \oplus \boldsymbol{F}_{2}$.

Since $\square: F_{1}^{s+2 r} \oplus F_{2}^{s+2 r} \rightarrow F_{1}^{s} \oplus F_{2}^{s}$ is isomorphic and $\square F_{1}^{s+2 r} \subset F_{1}^{s}, \square F_{2}^{s+2 r} \subset F_{2}^{s}$, we have $\square F_{i}^{s}=F_{i}^{s}, i=1,2$. Moreover, $\square F_{1}^{s+2 r}=A A^{*} F_{1}^{s+2 r}, \square F_{2}^{s+2 r}=B^{*} B F_{2}^{s+2 r}$. Since $A A^{*} F_{1}^{s+2 r} \subset A \Gamma^{s+r}\left(E_{-1}\right) \subset F_{1}^{s}$ and $B^{*} B F_{2}^{s+2 r} \subset B^{*} \Gamma^{s+r}\left(E_{1}\right) \subset F_{2}^{s}$, we have $A \Gamma^{s+r}\left(E_{-1}\right)=F_{1}^{s}, B^{*} \Gamma^{s+r}\left(E_{1}\right)=F_{2}^{s}$.

Since $A A^{*}: F_{1}^{s+r} \rightarrow F_{1}^{s-r}$ is isomorphic for $s \geqq 2 r$, we have that $A^{*} F_{1}^{s+r}$ is closed in $\Gamma^{s}\left(E_{-1}\right)$. This is because

$$
\|A\|\left\|A^{*} u\right\|_{s} \geqq\left\|A A^{*} u\right\|_{s-r} \geqq C\|u\|_{s+r},
$$

where $\|A\|$ is the operator norm of $A: \Gamma^{s}\left(E_{-1}\right) \rightarrow \Gamma^{s-r}\left(E_{0}\right)$. By the same method we get $B F_{2}^{s+r}$ is closed in $\Gamma^{s}\left(E_{1}\right)$.

COROllaRy 2. Let $\pi_{i}: \boldsymbol{\Gamma}_{\square}\left(E_{0}\right) \rightarrow \boldsymbol{F}_{i}, i=1,2$ be the projection. Then $\pi_{i}$ can be extended to the projection $\Gamma_{\square}^{s}\left(E_{0}\right)$ onto $F_{i}^{s}$ for $s \geqq 2 r$ and $\pi_{1}=\square^{-1} A A^{*}$, $\pi_{2}=\square^{-1} B^{*} B$.

COROLLARY 3. Let $\tilde{\pi}_{i}$ be the right invariant bundle map of $\gamma_{0}^{\text {t,s }}\left(E_{0}\right)$ into itself defined by $R_{\boldsymbol{g}}^{*} \pi_{i} R_{\boldsymbol{g}}^{*-1}$ for $t \geqq s \geqq n+5+2 r$. Then $\tilde{\pi}_{i}$ is a smooth projection.

Proof. Since $\tilde{\pi}_{1}=\widetilde{\square}^{-1} \widetilde{A A^{*}}, \quad \tilde{\pi}_{2}=\widetilde{\square}^{-1} B^{*} B$, Theorem A together with Lemma 5 yields the desired results.

Let $\tilde{F}_{1}^{t, s}=\operatorname{Ker}\left\{\tilde{\pi}_{2}: \gamma_{\square}^{t, s}\left(E_{0}\right) \rightarrow \gamma_{\square}^{t, s}\left(E_{0}\right)\right\}$ and $\tilde{F}_{2}^{t, s}=\operatorname{Ker}\left\{\tilde{\pi}_{1}: \gamma_{\square}^{t, s}\left(E_{0}\right) \rightarrow \gamma_{\square}^{t, s}\left(E_{0}\right)\right\}$. By Lemma 3, we see that $\widetilde{F}_{i}^{t, s}, i=1,2$, are smooth subbundle of $\gamma_{0}^{t, s}\left(E_{0}\right)$ for any $t \geqq s \geqq n+5+2 r$. Of course, $\tilde{\pi}_{i} \gamma_{\square}^{t, s}\left(E_{0}\right)=\tilde{F}_{i}^{t, s}$ and $\gamma_{0}^{t, s}\left(E_{0}\right)=\tilde{F}_{1}^{t, s} \oplus \tilde{F}_{2}^{t, s}$. (Whitney sum). Moreover, $\widetilde{F}_{1}^{t, s} \oplus \widetilde{F}_{2}^{t, s^{\prime}}$ is a smooth vector bundle over $\mathcal{D}_{0}^{t}$ for any $s, s^{\prime}$ such that $t \geqq s, s^{\prime} \geqq n+5+2 r$. Since $\gamma^{t, s}\left(E_{0}\right)=\gamma_{0}^{t, s}\left(E_{0}\right) \oplus \tilde{H}$, we can define the projection $\tilde{\pi}$ of $\gamma^{t, s}\left(E_{0}\right)$ onto $\tilde{F}_{1}^{t, s}$. Obviously, $\tilde{\pi}$ is smooth.

THEOREM 3. Notations and assumptions being as above, let $D, D^{\prime}$ be linear differential operators of order $p, q$ respectively, $p \geqq q, D$ and $D^{\prime}$ have smooth coefficients and map $\boldsymbol{\Gamma}\left(T_{M}\right)$ into $\boldsymbol{\Gamma}\left(E_{0}\right)$. Assume furthermore that $D \boldsymbol{\Gamma}\left(T_{M}\right) \subset \boldsymbol{F}_{1}$ and $\left(D+D^{\prime}\right) \Gamma^{s}\left(T_{M}\right)$ is closed in $F_{1}^{s-p} \oplus F_{2}^{3-q} \oplus H$ for any $s \geqq n+5+2 r+p$. Then, the kernel of $\tilde{D}+\widetilde{D}^{\prime}$ is a smooth subbundle of $\gamma^{t, s}\left(T_{M}\right)$, where $\widetilde{D}+\widetilde{D}^{\prime}$ is the right invariant bundle morphism defined by $R_{g}^{*}\left(D+D^{\prime}\right) R_{g}^{*-1}$. (This is a mapping of $\gamma^{t, s}\left(T_{M}\right)$ into $\tilde{F}_{1}^{t, s-p} \oplus \tilde{F}_{2}^{t, s-q} \oplus \tilde{H}$.)

PROOF. Since the closedness of the image is assumed, we have only to show the smoothness of $\widetilde{D}+\widetilde{D}^{\prime}$. Obviously, $\widetilde{D}+\widetilde{D}^{\prime}=\widetilde{D}+\tilde{\pi} \widetilde{D}^{\prime}+(1-\tilde{\pi}) \widetilde{D}^{\prime}$. Since $D \Gamma\left(T_{M}\right) \subset \boldsymbol{F}_{1}, \widetilde{D}+\tilde{\pi} \tilde{D}^{\prime}$ is a mapping of $\gamma^{t, s}\left(T_{M}\right)$ into $\tilde{F}_{1}^{t, s-p}$. By Theorem A and Lemma 5 , this is smooth. Similarly, $(1-\tilde{\pi}) \tilde{D}^{\prime}$ is smooth. So Lemma 3 shows that the kernel of $\widetilde{D}+\widetilde{D}^{\prime}$ is smooth.

This is an extension of Theorem 2, and these Theorems 2, 3 have several non-trivial applications. In the remaining part of this section, we discuss 
about the applications. There will be given four examples $A-D, A, B$ and $C$ are applications of Theorems 2-3 and D is an application of Theorem A and Lemma 5.

(A) Let $\boldsymbol{\Gamma}\left(\wedge_{M}^{k}\right)$ be the space of smooth $k$-forms on $M, \wedge_{M}^{k}$ the exterior product of cotangent bundle $T_{M}^{*}$. Suppose $M$ is odd dimensional $(n=2 m+1)$ and has a smooth contact structure. A contact structure of $M$ is given by a 1 -form $w$ such that $w \wedge(d w)^{m} \neq 0$. We define a smooth vector field $\xi_{w}$ by $\left.w\lrcorner \xi_{w} \equiv 1, d w\right\lrcorner \xi_{w} \equiv 0$ and smooth subbundles $\left.E_{w}=\left\{u \in T_{M} ; w\right\lrcorner u=0\right\}$, $\left.\hat{E}_{w}=\left\{u^{\prime} \in T_{M}^{*} ; u^{\prime}\right\lrcorner \xi_{w}=0\right\}$, where $\lrcorner$ implies the inner product. Since $w \wedge(d w)^{m}$ $\neq 0$, the mapping $d w: E_{w} \rightarrow \hat{E}_{w}$ defined by $\left.d w(u)=d w\right\lrcorner u$ is an isomorphism.

The Lie derivative of $w$ is given by

$$
\left.\left.\mathfrak{L}_{u} w=d(w\lrcorner u\right)+d w\right\lrcorner u \text {. }
$$

Since $T_{M}=R \xi_{w} \oplus E_{w}$ ( $R=$ the real numbers), any vector field $u$ of $M$ can be expressed by $f \xi_{w}+\hat{u}$, where $f$ is a function on $M, \hat{u}$ is a section of $E_{w}$. Using this expression, we have

$$
\left.\mathfrak{L}_{u} w=d f+d w\right\lrcorner \hat{u} .
$$

Let $1_{M}$ be the trivial bundle $R \times M$. In the above argument, replace $E_{-1}$ by $1_{M}, E_{0}$ by $T_{M}^{*}, E_{1}$ by $\wedge_{M}^{2}$ and $A, B$ by $d$. Then, we have $F_{1}^{s}=d \Gamma^{s+1}\left(1_{M}\right)$, $F_{2}^{s}=\delta \Gamma^{s+1}\left(\wedge_{M}^{2}\right)$ and $H=$ the space of harmonic 1 -forms. Let $\pi$ be the projection of $\Gamma\left(T_{M}^{*}\right)$ onto $F_{1}$. Then $\pi$ can be extended to the projection of $\Gamma^{s}\left(T_{M}^{*}\right)$ onto $F_{1}^{s}$.

Lemma 7. Assume $(1-\pi) d w\left(\Gamma^{s}\left(E_{w}\right)\right)$ is closed in $F_{2}^{s} \oplus H$. Then, the image $\left.\{d(w\lrcorner u)+d w\lrcorner u ; u \in \Gamma^{s}\left(T_{M}\right)\right\}$ is closed in $F_{1}^{3-1} \oplus F_{2}^{s} \oplus H$.

PROOF. It is not hard to verify that $\Gamma^{s}\left(T_{M}\right)=\Gamma^{s}\left(1_{M}\right) \xi_{w} \oplus \Gamma^{s}\left(E_{w}\right)$. Let $\left.\left.\operatorname{Im}^{s}=\{d(w\lrcorner u)+d w\right\lrcorner u ; u \in \Gamma^{s}\left(T_{M}\right)\right\}$. Suppose $x+y$ is in the closure of $\operatorname{Im}^{s}$ and $x \in F_{1}^{s-1}, y \in F_{2}^{s} \oplus H$. Then $y$ is contained in the closure of $(1-\pi) \mathrm{Im}^{s}$. By the assumption together with the fact $d w\left(\Gamma^{s}\left(E_{w}\right)\right)=d w\left(\Gamma^{s}\left(T_{M}\right)\right)$, we see that there is $u_{0} \in \Gamma^{s}\left(E_{w}\right)$ such that $\left.y=(1-\pi) d w\right\lrcorner u_{0}$. Since $x \in F_{1}^{s-1}=d \Gamma^{s}\left(1_{M}\right)$ and $\pi d w\lrcorner u_{0} \in F_{1}^{s-1}$, we can find $f \in \Gamma^{s}\left(1_{M}\right)$ such that $\left.d f=x-\pi d w\right\lrcorner u_{0}$. Therefore $\left.\left.d(w\lrcorner\left(f \xi_{w}+u_{0}\right)\right)+d w\right\lrcorner\left(f \xi_{w}+u_{0}\right)=x+y$. This implies $x+y \in \operatorname{Im}^{s}$. Thus, $\operatorname{Im}^{s}$ is closed in $F_{1}^{s-1} \oplus F_{2}^{s} \oplus H$.

THEOREM 4. Let

$$
\left.\left.\mathbb{S}=\left\{u \in \boldsymbol{\Gamma}\left(T_{M}\right) ; d(w\lrcorner u\right)+d w\right\lrcorner u=0\right\} .
$$

Assume $(1-\pi) d w\left(\Gamma^{s}\left(E_{w}\right)\right)$ is closed in $F_{2}^{s} \oplus H$ for any $s \geqq n+8$. Then for the subalgebra \& there is an I. L. H.-Lie subgroup $G$ which has \& as the Lie algebra.

Proof. In Theorem 3, put $\left.D u=d(w\lrcorner u), D^{\prime} u=d w\right\lrcorner u$. Then Lemma 7 shows that $D+D^{\prime}$ satisfies the conditions of Theorem 3. By using Proposition A and the properties $(G, 1-7)$, we have the desired result. 
Note: Put $\mathscr{D}_{w}=\left\{\varphi \in \mathscr{D}_{0} ; \varphi^{*} w=w\right\}, \mathscr{D}_{w}^{s}=\left\{\varphi \in \mathscr{D}_{0}^{s} ; \varphi^{*} w=w\right\}$. Then it is easy to see that $G \subset \mathcal{D}_{w}, G^{s} \subset \mathcal{D}_{w}^{s}$, because $G^{s}$ is given by the maximal integral submanifold of $\mathbb{S}^{s}$. Moreover, if $c(t)$ is a $C^{1}$-curve in $\mathscr{D}_{0}^{s}$ such that $c(t) \in \mathscr{D}_{w}^{s}$ for each $t$, then $c(t) \in G^{s}$. So, if we could prove $\mathscr{D}_{w}^{s}$ is locally $C^{1}$-arcwise connected, then $G^{s}$ and $\mathscr{D}_{w}^{s}$ coincide. However, the author could not prove that. In this case, we cannot use the same method as in [2], because the image Im $^{s}$ is closed in $F_{1}^{s-1} \oplus F_{2}^{s} \oplus H$, while $\varphi^{*} w$ moves in $F_{1}^{s-1} \oplus F_{2}^{s-1} \oplus H$. So the implicit function theorem cannot be applied.

(B) Suppose $M$ is even dimensional $(n=2 m)$ and have a smooth nondegenerate 2 -form $w$, where non-degenerate means $w^{m} \neq 0$. The Lie derivative of $w$ is given by

$$
\left.\left.\mathfrak{Q}_{u} w=d(w\lrcorner u\right)+d w\right\lrcorner u .
$$

In the argument of Lemma 5,6 and Theorem 3 , replace $E_{-1}$ by $T_{M}^{*}, E_{0}$ by $\wedge_{M}^{2}, E_{1}$ by $\wedge_{M}^{3}$ and $A, B$ by $d$. Then, we have $F_{1}^{s}=d \Gamma^{s+1}\left(T_{M}^{*}\right), F_{2}^{s}=\delta \Gamma^{s+1}\left(\wedge_{M}^{3}\right)$ and $H=$ the space of harmonic 2 -forms. Let $\pi$ be the projection of $\Gamma\left(\wedge_{M}^{2}\right)$ onto $\boldsymbol{F}_{1}$. Then $\pi$ can be extended to the projection of $\Gamma^{s}\left(\wedge_{M}^{2}\right)$ onto $F_{1}^{s}$ and that of $F_{1}^{s-1} \oplus F_{2}^{s} \oplus H$ onto $F_{1}^{s-1}$.

Assume the following two things:

(i) $\left.\{(1-\pi) d w\lrcorner u ; u \in \Gamma^{s}\left(T_{M}\right)\right\}$ is closed in $F_{2}^{s} \oplus H$ for any $s \geqq n+8$.

(ii) Let $\left.E^{s}=\left\{u \in \Gamma^{s}\left(T_{M}\right) ; d w\right\lrcorner u \in F_{1}^{s-1}\right\}$. Then $\left.F_{1}^{s-1}=\{d(w\lrcorner u) ; u \in E^{s}\right\}$. These assumptions are satisfied automatically, if $w$ is a symplectic 2 -form, that is, $d w=0$.

LEMMA 8. Notations and assumptions being as above, the space $\mathrm{Im}^{s}=$ $\left.\{d(w\lrcorner u)+d w\lrcorner u ; u \in \Gamma^{s}\left(T_{M}\right)\right\}$ is closed in $F_{1}^{s-1} \oplus F_{2}^{s} \oplus H$.

Proof. Put $\left.\left.T_{1} u=d(w\lrcorner u\right), T_{2} u=d w\right\lrcorner u$. Since $T_{1} \Gamma^{s}\left(T_{M}\right) \subset F_{1}^{s-1}, E^{s}$ is the kernel of $(1-\pi)\left(T_{1}+T_{2}\right): \Gamma^{s}\left(T_{M}\right) \rightarrow F_{2}^{s} \oplus H$. Obviously $(1-\pi)\left(T_{1}+T_{2}\right)=(1-\pi) T_{2}$.

Let $E_{0}^{s}$ be the kernel of $T_{1}: E^{s} \rightarrow F_{1}^{s-1}$ and $E_{1}^{s}$ the orthogonal complement of $E_{0}^{s}$ in $E^{s}$. By the assumption (ii), $T_{1}$ is an isomorphism of $E_{1}^{s}$ onto $F_{1}^{s-1}$. Consider the mapping $T_{1}^{-1}\left(T_{1}+T_{2}\right): E_{1}^{s} \rightarrow E_{1}^{s}$. Since $T_{2} E^{s} \subset F_{1}^{s-1}$ by definition, this is well defined and has the form $I+$ compact operator. This is because $T_{2} E^{s} \subset F_{1}^{s}$ and the inclusion $F_{1}^{s} \subset F_{1}^{s-1}$ is a compact operator by Rellich theorem. Thus, $\left(I+T_{1}^{-1} T_{2}\right) E_{1}^{s}$ is closed and finite codimensional and hence so does $\left(T_{1}+T_{2}\right) E_{1}^{s}$ in $F_{1}^{s-1}$. Therefore $\left(T_{1}+T_{2}\right) E^{s}$ is finite codimensional, because $\left(T_{1}+T_{2}\right) E^{s} \supset\left(T_{1}+T_{2}\right) E_{1}^{s}$ and hence closed in $F_{1}^{s-1}$.

Now, let $\left(T_{1}+T_{2}\right) u_{n}$ be a Cauchy sequence in $F_{1}^{s-1} \oplus F_{2}^{s} \oplus H$. Then, so is $(1-\pi) T_{2} u_{n}$ in $F_{2}^{s} \oplus H$. Let $E_{2}^{s}$ be the orthogonal complement of $E^{s}$ in $\Gamma^{s}\left(T_{M}\right)$. Then $(1-\pi) T_{2}: E_{2}^{s} \rightarrow F_{2}^{s} \oplus H$ is injective and has a closed image by the assumption (i). Therefore, there exists a Cauchy sequence $v_{n}$ in $E_{2}^{s}$ such that

$$
(1-\pi) T_{2} v_{n}=(1-\pi) T_{2} u_{n} .
$$


Since $v_{n}$ is a Cauchy sequence in $E_{2}^{s}, \lim _{n \rightarrow \infty} v_{n}=v_{0}$ exists in $E_{2}^{s}$. Therefore $(1-\pi) T_{2} v_{0}=\lim _{n \rightarrow \infty}(1-\pi) T_{2} u_{n}$. Obviously, $u_{n}-v_{n} \in E^{s}$, and $\left(T_{1}+T_{2}\right)\left(u_{n}-v_{n}\right)$ is a Cauchy sequence in $F_{1}^{s-1}$. On the other hand, by the above argument we see $\left(T_{1}+T_{2}\right) E^{s}$ is closed in $F_{1}^{s-1}$. Therefore, there is $x_{0} \in E^{s}$ such that $\left(T_{1}+T_{2}\right) x_{0}$ $=\lim _{n \rightarrow \infty}\left(T_{1}+T_{2}\right)\left(u_{n}-v_{n}\right)$. Thus, $\left(T_{1}+T_{2}\right) x_{0}=\lim _{n \rightarrow \infty}\left(T_{1}+T_{2}\right) u_{n}-\left(T_{1}+T_{2}\right) v_{0}$. This implies that $\lim _{n \rightarrow \infty}\left(T_{1}+T_{2}\right) u_{n}$ is contained in the image, that is, $\operatorname{Im}^{s}$ is closed.

THEOREM 5. Let $\left.\left.\mathbb{B}=\left\{u \in \Gamma\left(T_{M}\right) ; d(w\lrcorner u\right)+d w\right\lrcorner u=0\right\}$. Suppose $w$ satisfies the property (i) (ii) above. Then for the subalgebra $\mathbb{B}$ there is an I.L.H.-Lie subgroup $G$ which has $\&$ as the Lie algebra.

Note: Put $\mathscr{D}_{w}=\left\{\varphi \in \mathscr{D}_{0} ; \varphi^{*} w=w\right\}, \mathscr{D}_{w}^{s}=\left\{\varphi \in \mathscr{D}_{0}^{s} ; \varphi^{*} w=w\right\}$. Then $G \subset \mathscr{D}_{w}$ and $G^{s} \subset \mathscr{D}_{w}^{s}$. However, by the same reason as in (A), the author could not prove the equality. If $w$ is a symplectic two form, then we can use the implicit function theorem and hence $G=\mathscr{D}_{w}, G^{s}=\mathscr{D}_{w}^{s}$ (see [2]).

(C) Let $E$ be a smooth finite dimensional vector bundle over $M$. Suppose $E$ has a smooth riemannian connection $D$. Let $w$ be a smooth bundle morphism of $T_{M}$ into $E$ such that

$$
\nabla_{X} w(Y)-\nabla_{Y} w(X)-w([X, Y])=0,
$$

for any $C^{1}$-vector field of $M$. Remark that the image $w\left(T_{M}\right)$ is not necessarily closed or subbundle of $E$. This mapping $w$ can be regarded as a mapping of $\boldsymbol{\Gamma}\left(T_{M}\right)$ into $\boldsymbol{\Gamma}(E)$. By the assumption, $\mathbb{B}=\left\{u \in \boldsymbol{\Gamma}\left(T_{M}\right) ; w(u)=0\right\}$ is a subalgebra of $\boldsymbol{\Gamma}\left(T_{M}\right)$.

THEOREM 6. Notations and assumptions being as above, suppose $w \Gamma^{s}\left(T_{M}\right)$ is closed in $\Gamma^{s}(E)$ for any $s \geqq n+5$. Then, there is an I. L. H.-Lie subgroup $G$ of $\mathscr{D}_{0}$ which has $\mathbb{B}$ as the Lie algebra.

PRoOF. Since $w: \boldsymbol{\Gamma}\left(T_{M}\right) \rightarrow \boldsymbol{\Gamma}(E)$ is a differential operator of order 0 , Theorem $\mathrm{A}$ and Theorem 2 yield the desired results.

Note: Since $w\left(T_{M}\right)$ might not be a subbundle, the kernel of $w: T_{M} \rightarrow E$ might not be a subbundle of $T_{M}$.

The condition $\nabla_{X} w(Y)-\nabla_{Y} w(X)-w([X, Y])=0$ is used only to ensure that $\mathbb{B}$ is a subalgebra.

(D) Let $E$ be a smooth, riemannian and finite dimensional vector bundle over $M$. Let $A: \boldsymbol{\Gamma}(E) \rightarrow \boldsymbol{\Gamma}(E)$ be a self-adjoint elliptic differential operator of order $2 r$ with smooth coefficients such that all of the eigenvalues are $\leqq 0$. Then the resolvent $(p I-A)^{-1}$ exists for $p$ in the complement of $\Sigma$ in the complex plane and satisfies

$$
\left\|(p I-A)^{-1}\right\|_{s} \leqq \frac{C_{s}}{|p|+1},
$$

where $(p I-A)^{-1}$ is regarded as the operator of $\Gamma^{s}(E)$ into itself. 


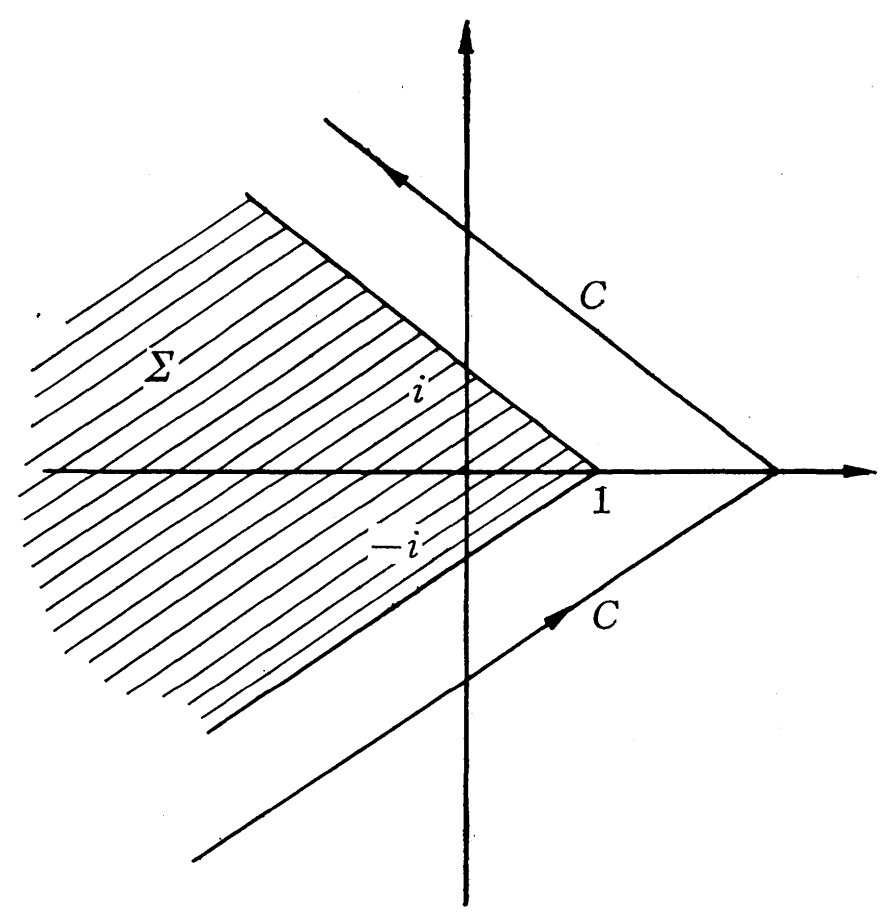

Fig.

Therefore, by Theorem 5.2 in $\left[10\right.$, p. 277], the operator $S_{t}$ of $\Gamma^{s}(E)$ into itself defined by

$$
S_{t} x=\frac{1}{2 \pi i} \int_{C} e^{p t}(p I-A)^{-1} x d p, \quad t>0, \quad S_{0}=i d
$$

is the semi-group with the generator $A$, where the route of the integral is as in Fig. Obviously, $R_{g}^{*} S_{t} R_{g}^{*-1}$ is a semi-group of the fibre of $\gamma^{t, s}(E)$ at $g$ with the generator $R_{g}^{*} A R_{g}^{*-1}$ for any $g \in \mathscr{D}_{0}^{t}$, where $t \geqq s \geqq n+5+2 r$. So define a bundle morphism $\widetilde{S}_{t}: \gamma^{t, s}(E) \rightarrow \gamma^{t, s}(E)$ by $R_{g}^{*} S_{t} R_{g}^{*-1}$. Then, we have

$$
\widetilde{S}_{t} u=\frac{1}{2 \pi i} \int_{C} e^{p t}(p I-\tilde{A})^{-1} u d p, \quad t>0,
$$

where $\tilde{A}$ is the bundle morphism of $\gamma^{t, s+2 r}(E)$ into $\gamma^{t, s}(E)$ and $(\not I-\tilde{A})^{-1}$ is regarded as the bundle morphism of $\gamma^{t, s}(E)$ into itself. It is possible because $\gamma^{t, s+2 r}(E) \subset \gamma^{t, s}(E)$.

By theorem A, $p I-\tilde{A}$ is a smooth bundle morphism of $\gamma^{t, s+2 r}(E)$ onto $\gamma^{t, s}(E)$. Therefore, Lemma 5 together with the fact that $\gamma^{t, s+2 r}(E) \subset \gamma^{t, s}(E)$ is smooth shows that $(p I-A)^{-1}$ is smooth.

Thus, we have the following

THEOREM 7. For any fixed $t \geqq 0, \widetilde{S}_{t}: \gamma^{t, s}(E) \rightarrow \gamma^{t, s}(E)$ is a smooth bundle morphism.

Note: By this theorem, we see that the semi-group used in $\S 13$ of [2] can be extended smoothly on the bundle $\gamma^{t, s}\left(T_{M}\right)$. 


\section{$3^{\circ}$ Jet bundles and higher order connections.}

Before starting to prove Theorem A and Lemma 2, we have to establish our notations and recall some facts which will be used in the next section. Specifically, we discuss $r$-th jet bundle of local $C^{r}$-maps of $M$ into itself. Let $I^{r}(M)$ be the jet bundle of local $C^{r}$-diffeomorphisms of $M$ into itself for $r \geqq 1$. Let $E$ be a smooth finite dimensional vector bundle over $M$ and $J^{r} E$ the $r$-th jet bundle of the local sections of $E$. In $1^{\circ}$, we made a vector bundle $\gamma(E)$. over $\mathscr{D}_{0}$ with the fibre $\Gamma(E)$. Correspondingly, in this section we will make a finite dimensional vector bundle $K^{r}(M, E)$ over $I^{r}(M)$ with the fibre $J_{x}^{r} E$, where $J_{x}^{r} E$ is the fibre of $J^{r} E$ at $x \in M$.

On the other hand, in [9], the author defined right invariant connections. on $\gamma\left(T_{M}\right)$, which was called I. L.H.-connections. Correspondingly, we will. define right invariant (in a sense) connections on $K^{r}(M, E)$. This is called a higher order connection or specifically $r$-th order connection. Such concepts. will be used to prove Theorem A, because we have to make a local trivialization of $\gamma\left(J^{r} E\right)$. Any differential operator $A$ of $\boldsymbol{\Gamma}(E)$ into $\boldsymbol{\Gamma}(F)$ with smooth coefficients is expressed by $A=\alpha j^{r}$ for some $\alpha \in \Gamma\left(\left(J^{r} E\right)^{*} \otimes F\right)$. So to prove$\tilde{A}$ is smooth, we have to show that (i) $\tilde{\alpha}: \gamma\left(J^{r} E\right) \rightarrow \gamma(F)$ is smooth and (ii). $\tilde{j}^{r}: \gamma(E) \rightarrow \gamma\left(J^{r} E\right)$ is smooth (see also next section for precise proof), where $\tilde{\alpha}, \tilde{j}^{r}$ are bundle morphisms defined by $R_{g}^{*} \alpha R_{g^{-1}}^{*}, R_{g}^{*} j^{r} R_{g^{-1}}^{*}$ respectively. In this section, we discuss about a local trivialization of $\gamma\left(J^{r} E\right)$.

Now, let $J^{r}(M)$ be the space of the $r$-th jets of the local $C^{r}$-mappings of $M$ into itself. $J^{r}(M)$ is a smooth fibre bundle over $M \times M$. Let $\pi: J^{r}(M) \rightarrow M \times M$ be the projection. Let $J^{r}(\{x\}, M)$ denote the full inverse $\pi^{-1}(\{x\} \times M)$. Suppose $a$ be an element of $J^{r}(M)$ with $\pi a=(x, y)$. Then, there is a smooth mapping $\varphi$ of a neighbourhood of $x$ into $M$ such that $\varphi(x)=y$ and $\left(j^{r} \varphi\right)(x)=a$.

Let $E$ be a smooth finite dimensional vector bundle over $M$. For the above $\varphi$, let $\varphi^{-1} E$ be the pull back of $E$ by $\varphi$. The local sections $v$ of $\varphi^{-1} E$ can be naturally identified with the mappings $v: M \rightarrow E$ such that $\pi^{\prime} v=\varphi$, where $\pi^{\prime}$ is the projection of $E$. Let $J_{x}^{r} \varphi^{-1} E$ be the vector space of the $r$-th jets at $x$ of the local sections of $\varphi^{-1} E$. If you use coordinates $x^{1}, \cdots, x^{n}$ of a neighborhood of $x$ and $y^{1}, \cdots, y^{n}, p^{1}, \cdots, p^{m}$ on an inverse image of $\pi^{\prime}$ of a neighborhood of $y$, then the mapping $v: M \rightarrow E$ can be expressed by

$$
\left(\varphi^{1}\left(x^{1}, \cdots, x^{n}\right), \cdots, \varphi^{n}\left(x^{1}, \cdots, x^{n}\right), p^{1}\left(x^{1}, \cdots, x^{n}\right), \cdots, p^{m}\left(x^{1}, \cdots, x^{n}\right)\right) .
$$

So $\left(j^{r} v\right)(x)=\left(\right.$ derivatives of $\varphi^{i}$ at $x$ up to order $r$, derivatives of $p^{i}$ at $x$ up. to order $r)$.

Therefore, we see $J_{x}^{r} \varphi^{-1} E$ depends only on $a \in J^{r}(M)$. So we denote this by $K_{a}^{r}(M, E)$. The same expression by coordinates gives the following:

LEMMA 9. $\hat{K}^{r}(M, E)=\bigcup\left\{K_{a}^{r}(M, E) ; a \in J^{r}(M)\right\}$ is a smooth vector bundle 
over $J^{r}(M)$.

Let $p$ be the projection of $\hat{K}^{r}(M, E)$ and $K^{r}(M, E)$ denote the restriction of $\widehat{K}^{r}(M, E)$ on $I^{r}(M)$ for $r \geqq 1$. Denote by $I^{r}(\{x\}, M), K^{r}(\{x\}, E)$ the restrictions of $I^{r}(M), K^{r}(M, E)$ on $\{x\} \times M, I^{r}(\{x\}, M)$ respectively.

Let $a \in J^{r}(M)$ and $\pi a=(x, y)$. Let $\hat{T}_{a} J^{r}(M)$ denote the vector space of the initial tangets of the curves $a(t)$ in $J^{r}(M)$ such that $a(0)=a, \pi a(t)=(x, y(t))$, that is, $a(t)$ fixes the point $x$. Another word $\hat{T}_{a} J^{r}(M)$ is the tangent space of $J^{r}(\{x\}, M)$ at $a$. So we see that $\hat{T} J^{r}(M)=\cup\left\{\hat{T}_{a} J^{r}(M) ; a \in J^{r}(M)\right\}$ is a smooth vector bundle over $J^{r}(M)$. Since $I^{r}(M)$ is an open subset of $J^{r}(M)$, we can define $\hat{T} I^{r}(M)$ by $\cup\left\{\hat{T}_{a} J^{r}(M) ; a \in I^{r}(M)\right\}$. Of course $\hat{T}_{a} J^{r}(M)$ is the tangent space of $I^{r}(\{x\}, M)$ at $a$.

LEMMA 10. $\widehat{T} J^{r}(M)=K^{r}\left(M, T_{M}\right)$.

PROOF. Let $\operatorname{Exp}_{z}: T_{z} M \rightarrow M$ be the exponential mapping of $M$ with respect to some smooth connection on $M$. Let $U$ be an element of $K_{a}^{r}\left(M, T_{M}\right)$. Put $\pi a=(x, y)$. There is a smooth mapping $\varphi$ of a neighbourhood of $x$ into $M$ such that $\left(j^{r} \varphi\right)(x)=a$, and there is a smooth section $v$ of $\varphi^{-1} T_{M}$ such that $\left(j^{r} v\right)(x)=U$. Let $\varphi_{t}(z)=\operatorname{Exp}_{z} t v(z)$. Then, $\left(j^{r} \varphi_{t}\right)(x)$ is an element of $J^{r}(M)$ such that $\left(j^{r} \varphi_{0}\right)(x)=a$. Therefore $\left.\frac{d}{d t}\right|_{t=0}\left(j^{r} \varphi_{t}\right)(x)$ is an element of $T J^{r}(M)$. It is easy to see that this does not depend on the choice of $\varphi, v$ as long as $\left(j^{r} \varphi\right)(x)=a,\left(j^{r} v\right)(x)=U$.

Conversely, let $X$ be the initial tanget of a curve $a(t)$ in $J^{r}(M)$ such that $a(0)=a, \pi a(t)=(x, y(t))$. Then, there is a smooth mapping $\varphi_{t}$ of a neighbourhood of $x$ into $M$ depending smoothly on $t$ such that $\left(j^{r} \varphi_{t}\right)(x)=a(t)$. For any $z$ in the neighborhood of $x$, define $v(z)$ by $v(z)=\left.\frac{d}{d t}\right|_{t=0} \varphi_{t}(z)$. Then, $v$ is identified with a smooth section of $\varphi_{0}^{-1} T_{M}$. It is easy to see that $X=\left.\frac{d}{d t}\right|_{t=0}\left(j^{r} \varphi_{t}\right)(x)=\left(j^{r} v\right)(x)$.

Let $U \in K^{r}(M, E)$ such that $p U=a, \pi a=(x, y)$. Let $\hat{T}_{U} K^{r}(M, E)$ denote the tangent space of $K^{r}(\{x\}, E)$ at $U$. Put $\widehat{T} K^{r}(M, E)=\cup\left\{\hat{T}_{U} K^{r}(M, E) ; U \in\right.$ $\left.K^{r}(M, E)\right\}$. Then $\hat{T} K^{r}(M, E)$ is a smooth vector bundle over $K^{r}(M, E)$ and in fact the subbundle of the tangent bundle of $K^{r}(M, E)$. Let $q: \hat{T} K^{r}(M, E)$ $\rightarrow K^{r}(M, E)$ be the projection.

Consider elements, $a, b \in I^{r}(M), v \in K^{r}(M, E), U^{\prime} \in \hat{T} K^{r}(M, E)$ such that $\pi a=(x, y), \pi b=(y, z), p U=b, q U^{\prime}=U$. We define right translations $R_{a} b$ (or simply $b a), R_{a}^{\prime} U, R_{a}^{\prime \prime} U^{\prime}$ by the following manner:

(i) Let $\varphi, \phi$ be local diffeomorphisms of neighborhoods of $x$ and $y$ into $M$ respectively such that $\left(j^{r} \varphi\right)(x)=a,\left(j^{r} \phi\right)(y)=b$. Define $R_{a} b$ by $\left(j^{r} \psi \varphi\right)(x)$. Of course $\pi R_{a} b=(x, z)$.

(ii) There is a local section $v$ of $\psi^{-1} E$ such that $\left(j^{r} v\right)(y)=U$. Since $v \varphi$ can be regarded as a local section of $\varphi^{-1} \psi^{-1} E$, we define $R_{a}^{\prime} U$ by $\left(j^{r} v \varphi\right)(x)$. 
Therefore, $R_{a}^{\prime} U \in K_{b a}^{r}(M, E)$.

(iii) There is a smooth curve $U(t)$ such that $U(0)=0,-\left.\frac{d}{d}\right|_{t=0} U(t)=U^{\prime}$ and $\pi p U(t)=(y, z(t))$. It is easy to see that $R_{a}^{\prime} U(t)$ is a smooth curve in $K^{r}(M, E)$. Define $R_{a}^{\prime \prime} U^{\prime}$ by $\left.\frac{d}{d t}\right|_{t=0} R_{a}^{\prime} U(t)$. Therefore $R_{a}^{\prime \prime} U^{\prime}$ is an element of $q^{-1} R_{a}^{\prime} U$.

As a matter of fact $R_{a}$ is a smooth diffeomorphism of $I^{r}(\{y\}, M)$ onto. $I^{r}(\{x\}, M), R_{a}^{\prime}$ a smooth bundle isomorphism of $K^{r}(\{y\}, M) \rightarrow K^{r}(\{x\}, M)$ such that the commutativity holds in the diagram

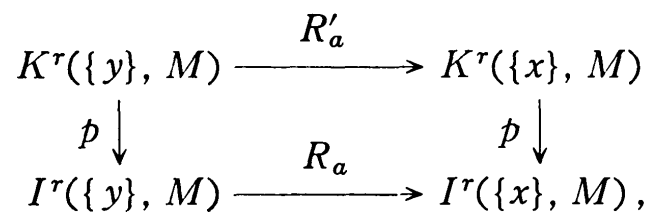

and $R_{a}^{\prime \prime}$ is the derivative of $R_{a}^{\prime}$.

Since $K^{r}\left(M, T_{M}\right)=\widehat{T} I^{r}(M), R_{a}^{\prime}$ and $R_{a}^{\prime \prime}$ are also defined on $\hat{T}^{r}(M)$ and $\hat{T} \hat{T} I^{r}(M)$ by the same manner, where $\hat{T} \hat{T} I^{r}(M)=\hat{T} K^{r}\left(M, T_{M}\right)$. A right invariant connection is a smooth subbundle of $\widehat{T} K^{r}(M, E)$ satisfying the following :

(1) For every $U \in K^{r}(M, E)$ such that $p U=b$, this subbundle is given by the image of linear mapping $\Gamma_{b}^{\prime}(*, U): \hat{T}_{b} I^{r}(M) \rightarrow \hat{T}_{U} K^{r}(M, E)$ such that $d p \Gamma_{b}^{\prime}(X, U) \equiv X$ for any $X \in \hat{T}_{b} I^{r}(M)$, where $d p$ is the derivative of the projection $p$.

(2) $\Gamma_{b}^{\prime}(X, U)$ is bi-linear with respect to $X, U$ as long as $U \in p^{-1} b$.

(3) Let $\pi b=(y, z)$. For any $a \in I^{r}(M)$ such that $\pi a=(x, y), R_{a}^{\prime \prime} \Gamma_{b}^{\prime}(X, U)=$ $\Gamma_{b a}^{\prime}\left(R_{a}^{\prime} X, R_{a}^{\prime} U\right)$.

If you restrict this subbundle on $\operatorname{Ir}^{r}(\{y\}, M)$, then this is one of smooth connections of $K^{r}(\{y\}, E)$ with right invariantness which comes from the condition (3). Conversely, if you have a smooth right invariant connection on $K^{r}(\{y\}, E)$ for some $y \in M$, then there exists uniquely a right invariant connection on $K^{r}(M, E)$. We have only to extend the connection on $K^{r}(\{y\}, E)$. by using (3). Such right invariant connections on $K^{r}(\{y\}, E)$ are the same with what Morimoto (c.f. his recent works, probably in Nagoya J. Math.) calls $r$-th order connections.

Anyway, by this definition, we can define a parallelism along a curve $a(t)$. such that $\pi a(t)=(x, y(t))$ (c.f. [12], p. 27). In case of $E=T_{M}$, a curve at $a(t)$, in $I^{r}(M)$ such that $\pi a(t)=(x, y(t))$ is called geodesic, if $\frac{d}{d t} a(t)$ is parallel along the curve $a(t)$. Since the condition $\pi a(t)=(x, y(t))$ implies that $a(t)$ is a curve in $I^{r}(\{x\}, M)$, such parallelism and the concept of geodesics are the same with the natural things on $K^{r}(\{x\}, M)$ or $I^{r}(\{x\}, M)$. 
Let $\nabla$ be a smooth connection on $E$. For any curve $c(t)$ on $M$, denote by $\tau(c(t)) v$ the parallel displacement of a vector $v \in \pi^{\prime-1} c(0)$ along a curve $c(t)$. $\tau(c(t)) v$ is an element of $\pi^{\prime-1} c(t)$. By using the connection $\nabla$ on $E$, we can define an $r$-th order right invariant connection $\Gamma^{\prime}$ on $K^{r}(M, E)$ by the following manner:

Let $X \in \hat{T}_{a} I^{r}(M)$ such that $\pi a=(x, y)$. Then, there is a curve $a(t)$ in $I^{t}(\{x\}, M)$ satisfying $a(0)=a,\left.\frac{d}{d t}\right|_{t=0} a(t)=X$. There exists a local diffeomorphism $\varphi_{t}$ of a neighbourhood of $x$ into $M$ such that $\left(j^{r} \varphi_{t}\right)(x)=a(t)$. Let $U \in K_{a}^{r}(M, E)=p^{-1} a$. Then, there is a local section $v$ of $\varphi_{0}^{-1} E$ such that $\left(j^{r} v\right)(x)=U$. This local section $v$ is identified with the mapping $v: M \rightarrow E$ such that $\pi^{\prime} v=\varphi_{0}$. Put $v_{t}(z)=\tau\left(\varphi_{t}(z)\right) v(z)$. Then, $v_{t}$ is a local section of $\varphi_{t}^{-1} E$. We define $\Gamma_{a}^{\prime}(X, U)$ by $\left.\frac{d}{d t}\right|_{t=0}\left(j^{r} v_{t}\right)(x)$. It is easy to see that $\left.\frac{d}{d t}\right|_{t=0}\left(j^{r} v_{t}\right)(x)$ depends only on $a, X, U$, because if you use a local coordinate and put $U_{t}=\left(j^{r} v_{t}\right)(x)$, then you get a differential equation that $U_{t}$ has to satisfy. The coefficients of that equation are depending only on $a(t)$, and $\left.\frac{d}{d t}\right|_{t=0} a(t)=X$, $U_{0}=U$. To get a more precise expression, we identify $v_{t}$ with the mapping $v_{t}: M \rightarrow E$ such that $\pi^{\prime} v_{t}=\varphi_{t}$. (Strictly speaking, the notation $v_{t}: M \rightarrow E$ is not good, because the domain of $v_{t}$ is a neighborhood of $x_{\text {.) }}$ ) So the derivative $\left.\frac{d}{d t}\right|_{t=0} v_{t}$ (this will be denoted by $u^{\prime}$ ) is a mapping of a neighborhood of $x$ into $T E$. It is easy to see that $\left(j^{r} u^{\prime}\right)(x)=\left.\frac{d}{d t}\right|_{t=0}\left(j^{r} v_{t}\right)(x)$.

For every $x \in M, e \in E$, let $\Gamma_{x}(*, e)$ be the linear mapping of $T_{x} M$ into $T E$ defined naturally from $\nabla$. This $\Gamma_{x}(*, e)$ gives a subbundle of $T E$ such that

(a) $d \pi^{\prime} \Gamma_{x}(u, e) \equiv u$ for any $u \in T_{x} M$,

(b) $\Gamma_{x}(u, e)$ is bi-linear with respect to $u, e$ as long as $e \in \pi^{\prime-1} x$.

Since $\nabla$ is a smooth connection, $\Gamma_{x}$ is smooth with respect to $x$. By using this notation, we have $u^{\prime}(z)=\Gamma_{\varphi_{0}(z)}(u(z), v(z))$, where $u(z)=\left.\frac{d}{d t}\right|_{t=0} \varphi_{t}(z)$. Thus, $\Gamma_{a}^{\prime}(X, U)=\left(j^{r} \Gamma_{\varphi_{0}}(u, v)\right)(x)$. Obviously, $\left(j^{r} \Gamma_{\varphi_{0}}(u, v)\right)(x)$ depends only on $a=$ $\left(j^{r} \varphi_{0}\right)(x), X=\left(j^{r} u\right)(x), U=\left(j^{r} v\right)(x)$.

It is easy to see that $\Gamma_{a}^{\prime}(X, U)$ satisfies the above condition (1), (2) and since $u^{\prime} \psi(z)=\Gamma_{\varphi_{0} \psi(z)}(u \psi(z), v \psi(z))$ for any local diffeomorphism $\psi$ of a neighborhood of $x^{\prime} \in M$ into $M$ such that $\phi\left(x^{\prime}\right)=x$, we see (3) is also satisfied.

Denote by $\tau^{r}(a(t)) U$ the parallel displacement of $U$ along the curve $a(t)$ with respect to the connection defined above. By the definition, we have the following :

LEMMA 11. For any $C^{1}$-curve $\varphi_{t}$ in $\mathscr{D}_{0}$ and $v \in \Gamma_{\varphi_{0}}(E)$, we have

$$
\tau^{r}\left(\left(j^{r} \varphi_{t}\right)(x)\right)\left(j^{r} v\right)(x)=\left(j^{r}\left(\tau\left(\varphi_{t}\right) v\right)\right)(x),
$$


where $\tau\left(\varphi_{t}\right) v$ is the parallel displacement of $\gamma(E)$ defined by $\left(\tau\left(\varphi_{t}\right) v\right)(z)=$ $\tau^{\prime}\left(\varphi_{t}(z)\right) v(z)$. Of course $\tau\left(\varphi_{t}\right) v$ is regarded as a mapping of $M$ into $E$ such that $\pi^{\prime} \tau\left(\varphi_{t}\right) v \equiv \varphi_{t}$.

Consider the case $E=T_{M}$. Let $\operatorname{Exp}^{r}$ denote the exponential mapping defined by the above connection. Of course $\operatorname{Exp}_{a}^{r}$ is a smooth mapping of an open neighbourhood of 0 of $\widehat{T}_{a} I^{r}(M)$ into $I^{r}(M)$.

LemMA 12. Let $\varphi \in \mathscr{D}_{0}$ and $u \in \Gamma_{\varphi}\left(T_{M}\right)$. Then $\operatorname{Exp}_{a}^{r}\left(j^{r} u\right)(x)=j^{r}\left(\operatorname{Exp}_{\varphi} u\right)(x)$, where $a=\left(j^{r} \varphi\right)(x)$ and $\operatorname{Exp}_{\varphi} u$ is a mapping defined by $\left(\operatorname{Exp}_{\varphi} u\right)(x)=\operatorname{Exp}_{\varphi(x)} u(x)$.

PROOF. Put $\varphi_{t}=\operatorname{Exp}_{\varphi} t u$. Then $\varphi_{t}(y)$ is geodesic in $M$ for each $y$, that is, $\frac{d}{d t} \varphi_{t}(y)=\tau^{\prime}\left(\varphi_{t}(y)\right) u(y)$. Applying $j^{r}$ to this equality and using Lemma 11 , we have

Therefore

$$
\frac{d}{d \bar{t}}\left(j^{r} \varphi_{t}\right)(x)=\tau^{r}\left(\left(j^{r} \varphi_{t}\right)(x)\right)\left(j^{r} u\right)(x)
$$

$$
\operatorname{Exp}_{a}^{r}\left(j^{r} u\right)(x)=\left(j^{r} \varphi_{t}\right)(x) .
$$

For any element $\varphi \in \mathscr{D}_{0}, \quad\left\{\left(j^{r} \varphi\right)(x) ; x \in M\right\}$ is a closed subset of $I^{r}(M)$. Denote this by $G^{r}(\varphi)$. By the definition of $K^{r}(M, E)$, it is easy to see that $K^{r}(M, E) \mid G^{r}(\varphi)=J^{r} \varphi^{-1} E$, where $\mid G^{r}(\varphi)$ implies the restriction to $G^{r}(\varphi)$. Let $U \in J_{x}^{r} \varphi^{-1} E$. Then, there is a smooth local section $v$ of $\varphi^{-1} E$ such that $\left(j^{r} \boldsymbol{v}\right)(x)=U$. Since $\varphi \in \mathscr{D}_{0}$, there is a smooth local section $v$ of $E$ at $\varphi(x)$ such that $v(z)=v \cdot \varphi(z)$. Recall the definition of the right translation. We have $\left(j^{r} v^{\prime}\right)(z)=R_{\left({ }^{r} \varphi\right)(x)}^{\prime}\left(j^{r} v\right)(\varphi(x))$. Since $\left(j^{r} v\right)(\varphi(x))$ can be regarded as an element of $\varphi^{-1} J^{r} E$, we have the following:

LEMMA 13. The right translation $R_{j r \varphi}^{\prime}$ defines a smooth isomorphism of $J^{r} \varphi^{-1} E$ onto $\varphi^{-1} J^{r} E$ for any $\varphi \in \mathscr{D}_{0}$.

Let $\operatorname{Exp}^{r} X$ denote the exponential mapping at $\left(j^{r} i d\right)(x)$ evaluated by $X \in J_{x}^{r} T_{M}$. If we take $u \in \Gamma\left(T_{M}\right)$ very small in $C^{1}$-uniform norm (c.f. $1^{\circ}$ ), then $\operatorname{Exp}^{r}\left(j^{r} u\right)(x)$ is an element of $I^{r}(M)$ for every $x \in M$. By Lemma 12, we have $\operatorname{Exp}^{r}\left(j^{r} u\right)(x)=\left(j^{r} \xi(u)\right)(x)$, where $\xi(u)(y)=\operatorname{Exp}_{y} u(y)$ (c. f. $1^{\circ}$ about this notation). Therefore, $\left\{\operatorname{Exp}^{r}\left(j^{r} u\right)(x) ; x \in M\right\}=G^{r}(\xi(u))$. Let $\tau^{r}\left(\operatorname{Exp}^{r}\left(j^{r} u\right)(x)\right) \tilde{v}(x)$ be the parallel displacement of $\tilde{v}(x)$ along the curve $\operatorname{Exp}^{r}\left(j^{r} u\right)(x), t \in[0,1]$, in $I^{r}(M)$. This is an element of $K_{a(x)}^{r}(M, E)$, where $a(x)=\operatorname{Exp}^{r}\left(j^{r} u\right)(x)$. Therefore, if we consider $x$ as a variable, then $\tau^{r}\left(\operatorname{Exp}^{r}\left(j^{r} u\right)(x)\right) \tilde{v}(x)$ gives a section of $K^{r}(M, E) \mid G^{r}(\xi(u))$, hence that of $J^{r} \xi(u)^{-1} E$. By using Lemma 13, we see that

$$
R_{\operatorname{Exp} r(j r u)(x)}^{\prime-1} \tau^{r}\left(\operatorname{Exp}^{r}\left(j^{r} u\right)(x)\right) \tilde{v}(x)
$$

is a section of $\xi(u)^{-1} J^{r} E$. We put

$$
\hat{\tau}^{r}(u, \tilde{v})(x)=R_{\operatorname{Exp} r(j r u)(x)}^{\prime-1} \tau^{r}\left(\operatorname{Exp}^{r}\left(j^{r} u\right)(x)\right) \tilde{v}(x) .
$$

$\hat{\boldsymbol{\tau}}^{r}(u, \tilde{v})$ is an element of $\boldsymbol{\Gamma}_{\xi(u)}(E)$. 
Assume $r \geqq 1$, because the case $r=0$ was already discussed (c.f. $1^{\circ}$ ). Recall the definition of the open subset $\hat{U}$ in $1^{\circ}$. This was a bounded open neighborhood of 0 of $\hat{\Gamma}^{1}\left(T_{M}\right)$ with $C^{1}$-uniform topology. Therefore, there is a relatively compact tubular neighborhood $W^{r}$ of the zero section of $J^{r} T_{M}$ such that the subset $\bar{U}^{s}$ of $\Gamma^{s}\left(T_{M}\right), s \geqq\left[\begin{array}{c}n \\ -2\end{array}\right]+1+r$, defined by $\dot{U}^{s}=\left\{u \in \Gamma^{s}\left(T_{M}\right)\right.$; $\left(j^{r} u\right)(x) \in W^{r}$, for every $\left.x \in M\right\}$ is contained in $\hat{U}$. (Here the assumption $r \geqq 1$ is used, and nothing more.) Put $\dot{\boldsymbol{U}}=\bar{U}^{s} \cap \boldsymbol{\Gamma}\left(T_{M}\right)$. Let $\hat{\tau}^{r}: \dot{\boldsymbol{U}} \times \boldsymbol{\Gamma}\left(J^{r} E\right) \rightarrow \gamma\left(J^{r} E\right)$ be the mapping defined by $\hat{\tau}^{r}(u, \tilde{v})$.

LEMMA 14. The above mapping $\hat{\tau}^{r}$ gives a local trivialization of $\gamma^{t, s}(E)$ for every $t, s$ such that $t \geqq s, t \geqq n+5+r, s \geqq n+5$.

Proof. Let $\tilde{\nabla}$ be a smooth connection of $J^{r} E$. (Since $J^{r} E$ is a smooth vector bundle over $M$, connections on $J^{r} E$ exist.) A local trivialization of $\gamma^{t, s}\left(J^{r} E\right)$ is given by using the parallel displacement $\tilde{\tau}$ defined by $\tilde{\nabla}$, namely, we define

$$
\tilde{\tau}(u, \tilde{v})(x)=\tilde{\tau}^{\prime}(\xi(u)(x)) \tilde{v}(x)
$$

as in $1^{\circ}$, where $\tilde{\tau}^{\prime}(\xi(u)(x))$ implies the parallel displacement along the geodesic $\xi(t u)(x), t \in[0,1]$. Let $\tilde{\tau}_{\xi(u)} \tilde{v}=\tilde{\tau}(u, \tilde{v})$. To prove this lemma, we have only to show that the mapping $\tilde{\tau}_{\bar{\xi}(u)}^{-1} \hat{\tau}^{r}(u, v)$ of $\dot{\boldsymbol{U}} \times \boldsymbol{\Gamma}\left(J^{r} E\right)$ into $\boldsymbol{\Gamma}\left(J^{r} E\right)$ can be extended to the $C^{\infty}$-mapping of $U^{t} \times \Gamma^{s}\left(J^{r} E\right)$ into $\Gamma^{s}\left(J^{r} E\right)$.

Let $p: J^{r} T_{M} \rightarrow T_{M}$ be the natural projection and $W^{r} \oplus J^{r} E$ the Whitney sum. We define a smooth mapping $\psi: W^{r} \oplus J^{r} E \rightarrow J^{r} E$ as following:

$$
\phi(X, V)=\tilde{\tau}^{\prime}(\operatorname{Exp} p X)^{-1} R_{\operatorname{Exp}^{r}{ }_{X}}^{-1} \hat{\tau}^{r}\left(\operatorname{Exp}^{r} X\right) V, \quad X \in J_{x}^{r} T_{M}, \quad V \in J_{x}^{r} E,
$$

where $\tilde{\tau}^{\prime}(\operatorname{Exp} p X), \tau^{r}\left(\operatorname{Exp}^{r} X\right)$ imply the parallel displacement along the curves $\operatorname{Exp} t p X, t \in[0,1]$, and $\operatorname{Exp}^{r} t X, t \in[0,1]$, respectively. Since $\tau^{r}\left(\operatorname{Exp}^{r} X\right) V$ is an element of $K_{a}^{r}(M, E), a=\operatorname{Exp}^{r} X$, we have that $R_{a}^{-1} \tau^{r}\left(\operatorname{Exp}^{r} X\right) V$ is an element of $J_{\operatorname{Exp} \pi_{X}}^{r} T_{M}$. To prove the smoothness, we have to use local coordinate and if we write all things by local coordinate, then the smoothness comes in naturally.

Let $J U^{s}=\left\{\tilde{u} \in \Gamma^{s}\left(J^{r} E\right) ; \tilde{u}(x) \in W^{r}\right.$ for any $\left.x \in M\right\}$. This is defined for $s \geqq\left[\frac{n}{2}\right]+1\left(\right.$ c. f. $\left.1^{\circ}\right)$. Then, letting $\Psi(\tilde{u}, \tilde{v})=\psi(\tilde{u}(x), \tilde{v}(x)), \Psi: J U^{s} \times \Gamma^{s}\left(J^{r} E\right) \rightarrow$ $\Gamma^{s}\left(J^{r} E\right)$ is smooth for $s \geqq n+5$, by using the fact mentioned in $2^{\circ}$. Obviously, $\Psi\left(j^{t} u, \tilde{v}\right)=\tilde{\tau} \bar{\xi}_{(u)}^{1} \tau^{r}(u, \tilde{v})$. Since $j^{r}: \Gamma^{t}\left(T_{M}\right) \rightarrow \Gamma^{t-r}\left(J^{r} T_{M}\right)$ is a bounded linear operator (c.f. next (c)), we see $j^{r}: \hat{U}^{t} \rightarrow J U^{t-r}$ is smooth for any $t \geqq\left[\frac{n}{2}\right]+1+r$. Clearly, $\tilde{U}^{t} \subset \hat{U}^{s}$ is smooth for any $t \geqq s$. Thus, we get $\tilde{\tau} \bar{\xi}(u) \tau(u, \tilde{v})$ is smooth.

Now, assume $E=T_{M}$. For any $X \in J_{x}^{r} T_{M},\left(d \operatorname{Exp}^{r}\right)_{X}$ can be regarded as a linear mapping of $J_{x}^{r} T_{M}$ into $\hat{T}_{\operatorname{Exp} r} J^{r}(M)$. In fact, if $X \in W^{r}$ (see just 
above Lemma 14), then $\left(d \operatorname{Exp}^{r}\right)_{X}: J_{x}^{r} T_{M} \rightarrow \widehat{T}_{\operatorname{Exp}^{r}{ }_{X}}(M)$ is isomorphic. By the same argument between Lemma 13 and Lemma 14, if $u \in \dot{U}, \tilde{v} \in \boldsymbol{\Gamma}\left(J^{r} T_{M}\right)$, then $\left(d \operatorname{Exp}^{r}\right)_{\left(j^{r} u\right)(x)} \tilde{v}(x)$ is a section of $K\left(M, T_{M}\right) \mid G^{r}(\xi(u))$, hence of $J^{r} \xi(u)^{-1} T_{M}$.

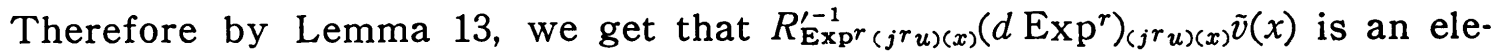
ment of $\boldsymbol{\Gamma}_{\xi(u)}\left(J^{r} T_{M}\right)$, where $x$ is regarded as a variable. Let $d \xi^{r}: \dot{U} \times \boldsymbol{\Gamma}\left(J^{r} T_{M}\right)$ $\rightarrow \gamma\left(J^{r} T_{M}\right)$ be the mapping defined by

$$
d \xi^{r}(u, \tilde{v})(x)=R_{\operatorname{Exp}^{r}(j r u)(x)}^{\prime-1}\left(d \operatorname{Exp}^{r}\right)_{(j r u)(x)} \tilde{v}(x) .
$$

LEMMA 15. $d \xi^{r}$ gives a local trivialization of $\gamma^{t, s}\left(J^{r} T_{M}\right)$ for every $t, s$ such that $t \geqq s, t \geqq n+5+r, s \geqq n+5$.

Proof. Define a mapping $\psi: W^{r} \oplus J^{r} T_{M} \rightarrow J^{r} T_{M}$ by

$$
\psi(X, Y)=\tilde{\tau}^{\prime}(\operatorname{Exp} p X)^{-1} R_{\operatorname{Exp}_{X}{ }_{X}}^{\prime-1}\left(d \operatorname{Exp}^{r}\right)_{X} Y, \quad X, Y \in J_{x}^{r} T_{M} .
$$

Then, the parallel argument as in Lemma 14, we have the desired results.

Proposition 1. Notations being as above, we have the following:

$$
\begin{aligned}
& d \xi^{r}\left(u, j^{r} v\right)=R_{\xi(u)}^{*} j^{r} R_{\xi(u)}^{*-1}(d \xi)_{u} v . \\
& \hat{\tau}^{r}\left(u, j^{r} v\right)=R_{\xi(u)}^{*} j^{r} R_{\xi(u)}^{*-1} \tau(u, v) .
\end{aligned}
$$

Especially, $\tilde{j}^{r}$ defined by $R_{\boldsymbol{g}}^{*} j^{r} R_{\boldsymbol{g}}^{*-1}$ is a smooth mapping of $\gamma^{s}\left(T_{M}\right)$ into $\gamma^{s, s-r}\left(J^{r} T_{M}\right)$ and $r^{s}(E)$ into $\gamma^{s, s-r}\left(J^{r} E\right)$ for any $s \geqq n+5+r$.

Proof. By Lemma 12, we have $\left(j^{r} \xi(u)\right)(x)=\operatorname{Exp}^{r}\left(j^{r} u\right)(x)$. So, we see $\left(j^{r}(d \xi)_{u} v\right)(x)=\left(d \operatorname{Exp}^{r}\right)_{\left({ }^{r} r u\right)(x)}\left(j^{r} v\right)(x)$. Therefore, letting $\varphi=\xi(u)$,

$$
\begin{aligned}
\left(j^{r} R_{\varphi-1}^{*}(d \xi)_{u} v\right)(x) & =R_{\left({ }^{r} \varphi^{-1}\right)(x)}^{\prime}\left(j^{r}(d \xi)_{u} v\right)\left(\varphi^{-1} x\right) \\
& =R_{\left(j \varphi^{-1}\right)(x)}^{\prime}\left(d \operatorname{Exp}^{r}\right)_{(j r u)\left(\varphi^{-1} x\right)}\left(j^{r} v\right)\left(\varphi^{-1} x\right) .
\end{aligned}
$$

Thus,

$$
\left(R_{\varphi}^{*} j^{r} R_{\varphi-1}^{*}(d \xi)_{u} v\right)(x)=R_{\left(j r \varphi^{-1}\right)(\varphi(x))}^{\prime}\left(d \operatorname{Exp}^{r}\right)_{(j r u)(x)}\left(j^{r} v\right)(x) .
$$

Since $R_{\left(j \varphi^{-1)}(\varphi(x))\right.}^{\prime}=R_{\left(j^{r} \varphi\right)(x)}^{\prime-1}$, we have

$$
R_{\xi(u)}^{*} j^{r} R_{\xi(u)}^{*-1}(d \xi)_{u} v=d \xi^{r}\left(u, j^{r} v\right) .
$$

On the other hand, we have

$$
\left(j^{r} R_{\varphi-1}^{*} \tau(u, v)\right)(x)=R_{\left(j r \varphi^{-1}\right)(x)}^{\prime}\left(j^{r} \tau(u, v)\right)\left(\varphi^{-1}(x)\right) .
$$

By Lemma 11-2, we have

So,

$$
\left(j^{r} R_{\varphi-1}^{*} \tau(u, v)\right)(x)=R_{\left(j \varphi_{\varphi^{-1}}^{\prime}(x)\right.}^{\prime r} \tau^{r}\left(\operatorname{Exp}^{r}\left(j^{r} u\right)\left(\varphi^{-1} x\right)\right)\left(j^{r} v\right)\left(\varphi^{-1} x\right) .
$$

$$
\begin{aligned}
\left(R_{\varphi}^{*} j^{r} R_{\varphi-1}^{*} \tau(u, v)\right)(x) & =R_{\left(j r \varphi^{-1}\right)(\varphi(x))}^{\prime r} \tau^{r}\left(\operatorname{Exp}^{r}\left(j^{r} u\right)(x)\right)\left(j^{r} v\right)(x) \\
& =\hat{\tau}^{r}\left(u, j^{r} v\right)(x) .
\end{aligned}
$$

These two equalities show that the local expression of $\tilde{j}^{r}$ is trivial, namely. 
if we put $d \xi_{u}^{r}(\tilde{v})=d \xi^{r}(u, \tilde{v})$, then $\left(d \xi_{u}^{r}\right)^{-1} \widetilde{j}^{r}(d \xi)_{u} v \equiv j^{r} v$, and, if we put $\hat{\tau}_{u}^{r}(v)=$ $\hat{\tau}^{r}(u, v)$, then $\left(\hat{\tau}_{u}^{r}\right)^{-1} \tilde{j}^{r} \tau(u, v) \equiv j^{r} v$.

\section{$4^{\circ}$ Proofs of Lemma 2, Theorem $A$ and several inequalities.}

(a) PROOF OF Lemma 2. Recall the definition of $U^{k_{0}+1}$ in the statement of Lemma 2. This was given by $\hat{U} \cap \Gamma^{k_{0+1}}\left(T_{M}\right)$ and $\hat{U}$ was a bounded open neighborhood of 0 of $\hat{\Gamma}^{1}\left(T_{M}\right)$ with $C^{1}$-uniform topology. Therefore, we may assume there is a relatively compact tubular neighborhood $W^{1}$ of zero section of $J^{1} T_{M}$ such that $\hat{U}=\left\{u \in \hat{\Gamma}^{1}\left(T_{M}\right) ; j^{1} u \in W^{1}\right\}$. Now, by the definition of $\hat{U}$, we may assume that $\operatorname{Exp}^{1} X$ is still contained in $I^{1}(M)$ for any $X \in W^{1}$, where $\operatorname{Exp}^{1}$ is the exponential mapping at $\left(j^{1} i d\right)(x), X \in J_{x}^{1} T_{M}$. Since $\operatorname{Exp}^{1} X$ is a 1 -jet of a local diffeomorphism, denoting by $\mu$ a volume element on $M$ we can define a mapping $J^{\prime}: W^{1} \rightarrow 1_{M}$ by the proportion of volume elements

$$
J^{\prime}(X)=\frac{1}{\mu(x)}\left(\operatorname{Exp}^{1} X\right) * \mu\left(\operatorname{Exp}_{x} p^{\prime} X\right),
$$

where $X=J_{x}^{1} T_{M}$ and $p^{\prime}$ is the natural projection of $J^{1} T_{M}$ onto $T_{M} . J^{\prime}$ is a smooth mapping. Therefore, by the fact in $1^{\circ}$ we see that $\hat{J}: \Gamma^{k_{0}}\left(W^{1}\right) \rightarrow$ $\Gamma^{k_{0}}\left(1_{M}\right)$ defined by $\hat{J}(u)(X)=J^{\prime}(\hat{u}(x))$ is a smooth mapping. On the other hand, $j^{1}: \Gamma^{k_{0+1}}\left(T_{M}\right) \rightarrow \Gamma^{k_{0}}\left(J^{1} T_{M}\right)$ is bounded linear, hence smooth. So together with the fact $J(u)=\hat{J}^{\circ} j^{1} u$, we see that $J: U^{k_{0}+1} \rightarrow \Gamma^{k_{0}}\left(1_{M}\right)$ is a smooth mapping.

(b) Proof of Theorem A. Let $E, F$ be vector bundles over $M$. Denote by $\tau^{\prime}(\operatorname{Exp} V), \tau^{\prime \prime}(\operatorname{Exp} V)$ the parallel displacements along the curve $\operatorname{Exp} t V$, $t \in[0,1]$, in $E$ and $F$ respectively, where $V$ is an element of the tangent bundle of $M$. Let $W^{r}$ be the same relatively compact tubular neighborhood of zero section of $J^{r} T_{M}$ as between Lemma 13 and 14. Let $\tau^{r}\left(\operatorname{Exp}^{r} X\right)$ be the parallel displacement along the curve $\operatorname{Exp}^{r} X, t \in[0,1]$ in $I^{r}(M)$, where $X \in J^{r} T_{M}$. .

Suppose $\alpha$ is an element of $\Gamma\left(\left(J^{r} T_{M}\right)^{*} \otimes F\right)$ or $\boldsymbol{\Gamma}\left(\left(J^{r} E\right)^{*} \otimes F\right)$, that is, $\alpha$ is a smooth section of $\left(J^{r} T_{M}\right)^{*} \otimes F$ or $\left(J^{r} E\right)^{*} \otimes F$. We define two smooth maps $\psi: W^{r} \rightarrow\left(J^{r} T_{M}\right)^{*} \otimes F, \psi^{\prime}: W^{r} \rightarrow\left(J^{r} E\right)^{*} \otimes F$ by the following :

$$
\begin{aligned}
& \phi(X)=\tau^{\prime \prime}(\operatorname{Exp} p X)^{-1} \alpha(\operatorname{Exp} p X) R_{\operatorname{Exp}^{\prime}{ }_{X}}^{\prime-1}\left(d \operatorname{Exp}^{r}\right)_{X} \\
& \phi^{\prime}(X)=\tau^{\prime \prime}(\operatorname{Exp} p X)^{-1} \alpha(\operatorname{Exp} p X) R_{\operatorname{Exp}^{r} X}^{\prime-1} \tau^{r}\left(\operatorname{Exp}^{r} X\right)
\end{aligned}
$$

where $p$ is the natural projection of $J^{r} T_{M}$ onto $T_{M}$.

Define the subsets $\dot{U}, U^{s}$ as just above Lemma 14, and let $\Gamma^{s}\left(W^{r}\right)=$ $\left\{\tilde{v} \in \Gamma^{s}\left(J^{r} T_{M}\right) ; \tilde{v}(x) \in W^{r}\right.$ for any $\left.x \in M\right\}$. Then, putting $\boldsymbol{\Gamma}\left(W^{r}\right)=\Gamma^{s}\left(W^{r}\right) \cap$ $\Gamma\left(J^{r} T_{M}\right)$, we have the following:

(i) Let $\Psi(v)(x)=\psi(v(x))$. Then $\Psi: \boldsymbol{\Gamma}\left(W^{r}\right) \rightarrow \boldsymbol{\Gamma}\left(\left(J^{r} T_{M}\right) * \otimes F\right)$ can be extended to the smooth mapping $\Psi: \Gamma^{s}\left(W^{r}\right) \rightarrow \Gamma^{s}\left(\left(J^{r} T_{M}\right)^{*} \otimes F\right)$ for any 
$s \geqq n+5 \quad$ (c. f. $)$.

(ii) Let $\Psi^{\prime}(\tilde{v})(x)=\psi^{\prime}(\tilde{v}(x))$. Then $\Psi: \boldsymbol{\Gamma}\left(W^{r}\right) \rightarrow \boldsymbol{\Gamma}\left(\left(J^{r} E\right)^{*} \otimes F\right)$ can be extended to the smooth mapping $\Psi^{\prime}: \Gamma^{s}\left(W^{r}\right) \rightarrow \Gamma^{s}\left(\left(J^{r} E\right) * \otimes F\right)$ for any $s \geqq n+5 \quad$ (c. f. $\$$ ).

Now, let $A: \boldsymbol{\Gamma}\left(T_{M}\right) \rightarrow \boldsymbol{\Gamma}(F)$ (or $A: \boldsymbol{\Gamma}(E) \rightarrow \boldsymbol{\Gamma}(F)$ ) be a differential operator of order $r$ with smooth coefficients. Then, there exists $\alpha \in \Gamma\left(\left(J^{t} T_{M}\right)^{*} \otimes F\right)$ (or $\boldsymbol{\Gamma}\left(\left(J^{r} E\right) * \otimes F\right)$ ) such that $A=a j^{r}$. Define a bundle morphism $\tilde{A}: \gamma\left(T_{M}\right) \rightarrow$ $\gamma(F)$ (or $\tilde{A}: \gamma(E) \rightarrow \gamma(F)$ ) by $R_{g}^{*} A R_{g}^{*}$. . To prove Theorem A, it is enough to show that $\Psi\left(j^{r} u\right) j^{r} v$ or $\Psi^{\prime}\left(j^{r} u\right) j^{r} v$ gives a local expression of $\tilde{A}$. Letting $\left(\tau_{\xi(u)}^{\prime \prime} v\right)(x)=\tau^{\prime \prime}(\operatorname{Exp} u(x)) v(x)$, the local expression of $\tilde{A}$ is given by $\tau_{\xi(u)}^{\prime \prime-1} \tilde{A}(d \xi)_{u} v$ or $\tau_{\xi(u)}^{\prime \prime-1} \tilde{A} \tau(u, v)$.

Put $\varphi=\xi(u)$. Then we have

$$
\begin{aligned}
\left(\Psi\left(j^{r} u\right) j^{r} v\right)(x) & =\psi\left(j^{r} u\right)(x)\left(j^{r} v\right)(x) \\
& =\tau^{\prime \prime}(\operatorname{Exp} u(x))^{-1} \alpha(\varphi(x)) R_{\operatorname{Exp}^{r}\left(j^{r} u\right)(x)}^{\prime-1} d\left(\operatorname{Exp}^{r}\right)_{\left(j^{r} u\right)(x)}\left(j^{r} v\right)(x) \\
& =\tau^{\prime \prime}(\operatorname{Exp} u(x))^{-1} \alpha(\varphi(x))\left(R_{\varphi}^{*} j^{r} R_{\varphi-1}^{*}(d \xi)_{u} v\right)(x) \\
& \quad(\text { c. f. Proposition 1) } \\
& =\tau^{\prime \prime}(\operatorname{Exp} u(x))^{-1}\left(R_{\varphi}^{*} \alpha j^{r} R_{\varphi-1}^{*}(d \xi)_{u} v\right)(x) \\
& =\left(\tau_{\xi(u)}^{\prime \prime-1} R_{\varphi}^{*} \alpha j^{r} R_{\varphi-1}^{*}(d \xi)_{u} v\right)(x) .
\end{aligned}
$$

On the other hand, by similar computations, we have

$$
\left(\Psi^{\prime}\left(j^{r} u\right) j^{r} v\right)(x)=\left(\tau_{\xi(u)}^{\prime \prime-1} R_{\varphi}^{*} \alpha j^{r} R_{\varphi-1}^{*} \tau(u, v)\right)(x) .
$$

Since $j^{r}: \Gamma^{s}\left(T_{M}\right) \rightarrow \Gamma^{s-r}\left(J^{r} T_{M}\right)$ (or, $\Gamma^{s}(E) \rightarrow \Gamma^{s-r}\left(J^{r} E\right)$ ) is bounded linear, the above computations complete the proof of Theorem A.

(c) Some inequalities. In (b), we get that the local expression of $\tilde{A}$ is given by $\Psi\left(j^{r} u\right) j^{r} v$ or $\Psi^{\prime}\left(j^{r} u\right) j^{r} v$. Here, in the last part of this paper, the norm $\left\|\Psi\left(j^{r} u\right) j^{r} v\right\|_{s}$ etc. will be calculated. Such estimations are needful to get a kind of regularity theorem. Inequalities given here will be used in next few papers.

By Lemma 13 in [9], we have

$$
\begin{aligned}
\left\|\Psi\left(j^{r} u\right) j^{r} v\right\|_{s}^{2} \leqq & e_{k_{0}}\left\{\left\|\Psi\left(j^{r} u\right)\right\|_{s}^{2}\left\|j^{r} v\right\|_{k_{0}}^{2}+\left\|\Psi\left(j^{r} u\right)\right\|_{k_{0}}^{2}\left\|j^{r} v\right\|_{s}^{2}\right\} \\
& +C_{s}\left\|\Psi\left(j^{r} u\right)\right\|_{s-1}^{2}\left\|j^{r} v\right\|_{s-1}^{2} .
\end{aligned}
$$

The same inequality holds for $\Psi^{\prime}$, where $k_{0}=\left[\begin{array}{c}n \\ 2\end{array}\right]+1, e_{k_{0}}$ a constant depending only on $k_{0}$ and $C_{s}$ is a constant depending on $s$.

By Theorem $\mathrm{A}$ in [9], we have the following inequality:

$$
\left\|\Psi\left(j^{r} u\right)\right\|_{s}^{2} \leqq P^{\prime}\left(\left\|j^{r} u\right\|_{2 k_{0}}^{2}\right)\left\|j^{r} u\right\|_{s}^{2}+Q_{s}^{\prime}\left(\left\|j^{r} u\right\|_{s-1}^{2}\right)
$$


where $P^{\prime}$ is a positive coefficients polynomial independent from $s$ and $Q_{s}^{\prime}$ is a polynomial depending on $s$. The same inequality holds for $\Psi^{\prime}$.

Let $(d \Psi)_{j r u} j^{r} v$ be the derivative of $\Psi$ at $j^{r} u$ evaluated by $j^{r} v$.

Then

$$
\Psi\left(j^{r} u\right)-\Psi(0)=\int_{0}^{1}(d \Psi)_{j} r_{\theta u} j^{r} u d \theta
$$

Therefore,

$$
\left\|\Psi\left(j^{r} u\right)-\Psi(0)\right\|_{s}^{2} \leqq \int_{0}^{1}\left\|(d \Psi)_{j^{r} \theta u} j^{r} u\right\|_{s}^{2} d \theta
$$

using Schwartz's inequality. Since $d \Psi$ (or $d \Psi^{\prime}$ ) is defined from smooth function $d \psi$ (or $d \psi^{\prime}$ ), we have the following inequality using Theorem $\mathrm{A}$ in [9]:

$$
\left\|\Psi\left(j^{r} u\right)-\Psi(0)\right\|_{s}^{2} \leqq P^{\prime \prime}\left(\left\|j^{r} u\right\|_{2 k_{0}}^{2}\right)\left\|j^{r} u\right\|_{s}^{2}+Q^{\prime \prime}\left(\left\|j^{r} u\right\|_{s-1}^{2}\right)\left\|j^{r} u\right\|_{s-1}^{2}
$$

where $P^{\prime \prime}$ is a positive coefficient polynomial independent from $s$ and $Q_{s}^{\prime \prime}$ is a polynomial depending on $s$. The same inequality holds for $\Psi^{\prime}$, where $s \geqq \operatorname{dim} M+5$.

LEMMA 16.

$$
\left\|j^{r} u\right\|_{s}^{2} \leqq 3^{r}\|u\|_{s+r}^{2}+C_{r}\|u\|_{s+r-1}^{2} .
$$

PROOF. First of all, we prove the following inequality:

$$
\left\|j^{1} u\right\|_{s}^{2} \leqq 3\|u\|_{s+1}^{2}+\|u\|_{s}^{2} .
$$

Consider the vector bundle $J^{1} T_{M}$ or $J^{1} E$. If we restrict the transition function of this bundle, then this can be naturally identified with $T_{M} \oplus T_{M} \otimes T_{M}$ or $E \oplus T_{M} \otimes E$. Another word, both vector bundle have transition functions which can be restricted to a common transition group. So by using a basis contained in this common transition group, two vector bundles can be naturally identified. In this sense, we can put $j^{1} u=\nabla u+u$. Therefore,

$$
\left\|j^{1} u\right\|_{s}^{2} \leqq\|\nabla u\|_{s}^{2}+\|u\|_{s}^{2}+2\|\nabla u\|_{s}\|u\|_{s} .
$$

Therefore, $\left\|j^{1} u\right\|_{s}^{2} \leqq 3\|u\|_{s+1}^{2}+\|u\|_{s}^{2}$. Using this inequality successively we get a desired one, because $j^{1} j^{k} u=j^{k+1} u$.

Combining inequalities $(4,5)$, Lemma 16 and the argument in (b), we get

PROPOSITION 2. Suppose $s \geqq n+5+r$. If $u$ is restricted in a bounded neighbourhood of 0 in $\Gamma^{2 k_{0}+r}\left(T_{M}\right)$, then the following inequalities hold:

$$
\begin{aligned}
& \left.\| \tau_{\xi(u)}^{\prime \prime-1}\right) \\
& \quad \tilde{A}(d \xi)_{u} v \|_{s}^{2} \\
& \quad \leqq C_{1}\|u\|_{s+r}^{2}\|v\|_{k_{0}+r}^{2}+C_{2}\|v\|_{s+r}^{2}+R_{s}\left(\|u\|_{s+r-1}^{2}\right)\|v\|_{s+r-1}^{2}, \\
& \| \tau_{\xi(u)}^{\prime \prime-1} \\
& \quad \tilde{A}(d \xi)_{u} v-A v \|_{s}^{2} \\
& \quad \leqq C_{1}^{\prime}\|u\|_{s+r}^{2}\|v\|_{k_{0}+r}^{2}+C_{2}^{\prime}\|u\|_{0_{0+r}}^{2}\|v\|_{s+r}^{2}+R_{s}^{\prime}\left(\|u\|_{s+r-1}^{2}\right)\|u\|_{s+r-1}^{2}\|v\|_{s+r-1}^{2} .
\end{aligned}
$$

The same inequalities hold for $\tau_{\xi(u)}^{\prime \prime-1} \tilde{A} \tau(u, v)$ and $\tau_{\xi(u)}^{-1} \tilde{A} \tau(u, v)-A v$, where $C_{1}, C_{2}$, 
$C_{1}^{\prime}, C_{2}^{\prime}$ are constants independent from $s$ and $R_{s}, R_{s}^{\prime}$ are polynomials depending on $s$.

\section{References}

[1] J. Dieudonne, Foundations of Modern analysis, Academic Press, 1960.

[2] D. Ebin and J. Marsden, Groups of diffeomorphisms and the motion of an in. compressible fluid, Ann. of Math., 92 (1970), 102-163.

[3] S. Lang, Introduction to differentiable manifolds, Interscience Publ. Inc., 1962.

[4] J. Leslie, On a differentiable structure of the group of diffeomorphisms, Topo. logy, 6 (1967), 263-271.

[5] J. Leslie, Some Frobenius theorem in global analysis, J. Differential Geometry, 2 (1968), 279-298.

[6] H. Omori, Homomorphic images of Lie groups, J. Math. Soc. Japan, 18 (1966), 97-117.

[7] H. Omori, On the group of diffeomorphisms on a compact manifold, Proc. Symp. Pure Math., vol XV, Amer. Math. Soc., 1970, 167-183.

[8] H. Omori, Regularity of connections, Diff. Geom. in honor of K. Yano, Kinokuniya, Tokyo, 1972, 385-399.

[9] H. Omori, Local structures of group of diffeomorphisms, J. Math. Soc. Japan, 24 (1972), 60-88.

[10] S. Mizohata, Theory of partial differential equations, Iwanami, 1965 (In Japanese).

[11] D. Montgomery and L. Zippin, Topological transformation groups, Interscience, New York, 1955.

[12] K. Nomizu, Lie groups and differential geometry, Publ. Math. Soc. Japan, 1965.

[13] R.S. Palais, Seminar on the Atiyah Singer index theorem, Princeton Study 57, 1965. 